\title{
Combinatorial Effects of Alpha- and Gamma-Protocadherins on Neuronal Survival and Dendritic Self-Avoidance
}

\author{
Samantha Ing-Esteves, ${ }^{1}$-Dimitar Kostadinov, ${ }^{2}$ Julie Marocha, ${ }^{1}{ }^{\circledR}$ Anson D. Sing, ${ }^{1}$ Kezia S. Joseph, ${ }^{1}$ \\ Mallory A. Laboulaye, ${ }^{2}$ ㅇoshua R. Sanes, ${ }^{2 *}$ and ${ }^{-}$Julie L. Lefebvre ${ }^{1,2 *}$ \\ ${ }_{1}$ Program for Neurosciences and Mental Health, Hospital for Sick Children, Department of Molecular Genetics, University of Toronto, Toronto, Ontario \\ M5G 0A4, Canada and 2Department of Molecular and Cellular Biology and Center for Brain Science, Harvard University, Cambridge, Massachusetts 02138
}

The clustered protocadherins (Pcdhs) comprise 58 cadherin-related proteins encoded by three tandemly arrayed gene clusters, $P c d h-\alpha$, $P c d h-\beta$, and $P c d h-\gamma(P c d h a, P c d h b$, and $P c d h g$, respectively). Pcdh isoforms from different clusters are combinatorially expressed in neurons. They form multimers that interact homophilically and mediate a variety of developmental processes, including neuronal survival, synaptic maintenance, axonal tiling, and dendritic self-avoidance. Most studies have analyzed clusters individually. Here, we assessed functional interactions between $P c d h a$ and $P c d h g$ clusters. To circumvent neonatal lethality associated with deletion of $P c d h g s$, we used Crispr-Cas9 genome editing in mice to combine a constitutive $P c d h a$ mutant allele with a conditional $P c d h g$ allele. We analyzed roles of Pcdhas and Pcdhgs in the retina and cerebellum from mice (both sexes) lacking one or both clusters. In retina, Pcdhgs are essential for survival of inner retinal neurons and dendritic self-avoidance of starburst amacrine cells, whereas Pcdhas are dispensable for both processes. Deletion of both $P c d h a$ and $P c d h g$ clusters led to far more dramatic defects in survival and self-avoidance than $P c d h g$ deletion alone. Comparisons of an allelic series of mutants support the conclusion that Pcdhas and Pcdhgs function together in a dose-dependent and cell-type-specific manner to provide a critical threshold of Pcdh activity. In the cerebellum, Pcdhas and Pcdhgs also cooperate to mediate self-avoidance of Purkinje cell dendrites, with modest but significant defects in either single mutant and dramatic defects in the double mutant. Together, our results demonstrate complex patterns of redundancy between Pcdh clusters and the importance of Pcdh cluster diversity in postnatal CNS development.

Key words: apoptosis; dendrite; neurite development; protocadherin; retina; self-avoidance

Significance Statement

The formation of neural circuits requires diversification and combinatorial actions of cell surface proteins. Prominent among them are the clustered protocadherins (Pcdhs), a family of $\sim 60$ neuronal recognition molecules. Pcdhs are encoded by three closely linked gene clusters called $P c d h-\alpha, P c d h-\beta$, and $P c d h-\gamma$. The Pcdhs mediate a variety of developmental processes, including neuronal survival, synaptic maintenance, and spatial patterning of axons and dendrites. Most studies to date have been limited to single clusters. Here, we used genome editing to assess interactions between $P c d h-\alpha$ and $P c d h-\gamma$ gene clusters. We examined two regions of the CNS, the retina and cerebellum and show that the $14 \alpha$-Pcdhs and $22 \gamma$-Pcdhs act synergistically to mediate neuronal survival and dendrite patterning.

\section{Introduction}

The complexity of neural circuits in the CNS implies a corresponding complexity of molecular mechanisms to pattern pro-

Received Oct. 20, 2017; revised Jan. 12, 2018; accepted Jan. 29, 2018.

Author contributions: J.R.S. and J.L.L. designed research; S.I.-E., D.K., J.M., A.D.S., K.S.J., M.A.L., and J.L.L. performed research; S.I.E., D.K., J.M., A.D.S., K.S.J., and J.L.L. analyzed data; J.R.S. and J.L.L. wrote the paper.

This work was supported by an Ontario Graduate Scholarship and a Vision Science Research Program Studentship from the University of Toronto (S.I.-E); a University of Toronto Open Fellowship (J.M.); a Restracomp Postdoctoral Fellowship from the Hospital for Sick Children (A.D.S.); a Canada Research Chair (Tier 2), a Sloan Fellowship in Neuroscience, an NSERC Discovery Grant (RPIN-2016-06128), the Hospital for Sick Children (J.L.L.); and the National Institutes of Health (Grants T32 EY007110 and F31 NS078893 to D.K. and Grant R01 EY022073 to J.R.S.). We thank Tom Maniatis and Weisheng Chen (Columbia University) for sharing the $P c d h a^{\mathrm{ko}}, P_{c d h} b^{\mathrm{ko}}$, and $P c d h a^{\text {mcherry }}$ mice jections and connections. An attractive hypothesis is that large families of recognition molecules underlie this specificity, with combinatorial expression of family members endowing neurons 
with distinct identities, patterning their axons and dendrites and biasing synapse formation in favor of appropriate partners (Zipursky and Sanes, 2010).

Candidate mediators of such processes include the clustered protocadherins (Pcdhs), a set of $\sim 60$ cadherin-related transmembrane molecules encoded by a tandem array of genes. $P c d h$ genes are subdivided into $P c d h-\alpha(P c d h a), P c d h-\beta(P c d h b)$, and $P c d h-\gamma$ (Pcdhg) clusters, encoding 14, 22, and 22 isoforms, respectively, in mice (see Fig. 1A) (Wu and Maniatis, 1999). Most isoforms engage in strict homophilic binding in cell aggregation assays (Schreiner and Weiner, 2010; Thu et al., 2014). Isoforms from the same and different clusters form cis-dimers, which interact homophilically in trans in anti-parallel arrangements (Nicoludis et al., 2015; Rubinstein et al., 2015; Rubinstein et al., 2017). Pcdhs are expressed throughout the nervous system and expression is combinatorial and stochastic in at least some populations (Esumi et al., 2005; Kaneko et al., 2006; Toyoda et al., 2014; Mountoufaris et al., 2017). Therefore, Pcdhs are well suited to mediate complex, specific interactions among neurons.

Initial attempts to analyze Pcdh function used genetic methods to inactivate individual clusters. Pcdhg cluster mutations resulted in degenerative defects in the spinal cord and neonatal lethality (Wang et al., 2002). Subsequent studies using conditional Pcdhg mutants and hypomorphs documented neuronal death in several regions, as well as defects in synaptic maintenance and dendritic patterning (Weiner et al., 2005; Lefebvre et al., 2008; Prasad et al., 2008; Garrett and Weiner, 2009; Schreiner and Weiner, 2010; Su et al., 2010; Garrett et al., 2012; Lefebvre et al., 2012; Kostadinov and Sanes, 2015; Molumby et al., 2016). Inactivation of the Pcdha cluster resulted in abnormal axonal arborizations in several brain areas (Hasegawa et al., 2008; Katori et al., 2009; Meguro et al., 2015; Chen et al., 2017; Mountoufaris et al., 2017).

A second set of studies used loss- and gain-of-function strategies to assay roles of individual isoforms. Three noteworthy sets of results have been reported. The first distinguished two seemingly contradictory behaviors of retinal starburst amacrine cells (SACs). Dendrites of individual SACs repel each other in a process called self-avoidance, leading to even coverage of their receptive field. Simultaneously, however, SAC dendrites also synapse with dendrites of neighboring SACs, implying that they are capable of self-/non-self-discrimination. We showed that single Pcdhg isoforms can mediate self-avoidance, but multiple isoforms are required for self-/non-self-discrimination (Lefebvre et al., 2012; Kostadinov and Sanes, 2015). Second, whereas any Pcdhg isoform is sufficient for self-avoidance, neuronal survival in retina and spinal cord is dependent on the PcdhgC3-C5 isoforms (Chen et al., 2012). Third, patterning of serotonergic axons is dependent on a single Pcdha-C2 isoform (Chen et al., 2017).

A third level of complexity involves interactions among $P c d h$ gene clusters. Recently, two groups deleted the entire $P c d h$ locus in mice, revealing cooperation among clusters in patterning of olfactory axons and survival of spinal neurons (Hasegawa et al., 2016; Mountoufaris et al., 2017). In their alleles, the neonatal lethality of the $P c d h g$ mutation restricted analysis to embryos. In an independent approach, we have generated a mutant allele $(P c d h-a g$ f $)$ in which constitutive Pcdha deletion is combined with conditional $P c d h g$ inactivation, circumventing neonatal lethality. Here, we compare phenotypes in single Pcdha, Pcdhb, and Pcdhg cluster mutants with those of $P c d h$ - ag ${ }^{\mathrm{f}}$ mutants, focusing on neuronal survival in retina and dendritic self-avoidance in retina and cerebellum. In retina, Pcdhas and Pcdhbs are dispensable on their own, but Pcdh-ag mutants dramatically enhance Pcdhg phenotypes, a phenomenon called unequal redundancy (Briggs et al.,
2006). In contrast, Purkinje cells are equally dependent on Pcdha and $P c d h g$ clusters for dendrite patterning, exhibiting partial redundancy. Together, the combined activities and diversity provided by the Pcdh clusters ensure robust neuronal patterning.

\section{Materials and Methods}

Mice. To generate $P c d h-a g^{\mathrm{f}}$ mutants, a single guide RNA (sgRNA) targeting the Pcdha constant exon 1 was designed using a CRISPR design tool (http://crispr.mit.edu/). Oligo duplexes were annealed into pX330-U6Chimeric_BB-CBh-hSpCas9, a gift from Feng Zhang (Addgene, https://www.addgene.org/search/advanced/?q=42230; Cong et al., 2013). Multiple sgRNAs were screened in HEK293 cells for cleavage of target DNA sequence and disruption of Pcdha-exon1-GFP reporter expression. sgRNA for target 5'-CGCTAAGAGCAGGCATGCAC-3' was transcribed in vitro from pX330 with a T7 RNA polymerase (Ambion) and injected with Cas9 RNA (Systems Biosciences) into Pcdh-g ${ }^{\text {fcon3 }} \mathrm{zy}$ gotes at the Harvard University Genome Modification Facility (https:// gmf.fas.harvard.edu, RRID:SCR_009849). Of 220 zygotes injected, 16 pups were born and 11 pups had insertion or deletions in Pcdha constant exon $1\left(P c d h a^{\text {conexldel }}\right)$. Founders were identified by amplifying the targeted genomic region with primers, forward: 5' TTGGGCAGGGCACAT CTAAG-3'; reverse: 5'-TCGTCAGTGGGAGATAGGCA-3', digesting with SphI which flanked the sgRNA target, and Sanger sequencing. Mating demonstrated that seven of the $P c d h a^{\text {conexldel }}$ mutations were on the same chromosome as the $P c d h-g^{\text {fcon3 } 3}$ allele and these lines were studied further $\left(P c d h-a g^{\mathrm{f}}\right)$. The $a g$ deletions removed most of the Pcdha constant exon 1 and the subsequent exon-intron junction and splice donor site. The deletions are predicted to disrupt splicing between constant exons 1 and 2 and to cause frame shift and premature stop codons in altered exon1-intron transcripts or in exon 2 in the event of exon skipping. The above primers were used for genotyping, and distinguish the WT (756 bp) and the deleted alleles: $a g^{\mathrm{f}}-1,623 \mathrm{bp}$, corresponding to a $133 \mathrm{bp}$ deletion; $a g^{\mathrm{f}}-2,637 \mathrm{bp}$, corresponding to a $119 \mathrm{bp}$ deletion; and $a g^{\mathrm{f}}-3$, $439 \mathrm{bp}$, corresponding to a $321 \mathrm{bp}$ deletion. The C-terminal Pcdha intracellular proteins encoded by constant exons 1-3 were not detected in Western blots of homozygous $a g^{\mathrm{f}}-1, a g^{\mathrm{f}}-2$, and $a g^{\mathrm{f}}-3$ brain and retina tissue, confirming that the deletions disrupted Pcdha protein production. For the studies reported here, we used the $P c d h-a g^{\mathrm{f}}$ lines $a g^{\mathrm{f}}-1$ and $a g^{\mathrm{f}}-3$ interchangeably.

Other lines have been described previously. The $P c d h a^{\text {mCherry }}$ reporter allele, in which the $m$ Cherry gene is fused in frame with the $P c d h a$ coding sequence at the $\mathrm{C}$ terminus, and the $P c d h a^{\mathrm{ko}}$ and $P c d h b^{\mathrm{ko}}$ alleles, in which the entire $P c d h a$ or $P c d h b$ clusters are inactivated, were described by Chen et al. (2017). The Pcdhg fcon3 conditional allele contains loxP sequences flanking the third constant exon and generates a functionally null allele after Cre recombination (Lefebvre et al., 2008; Prasad et al., 2008). The Six3-cre line expresses Cre in retinal progenitor cells, leading to inactivation throughout the central retina, but sparing peripheral regions (https://www.jax.org/strain/019755; RRID:IMSR_JAX:019755) (Furuta et al., 2000). Chat-cre knock-in mice express Cre in cholinergic neurons (https://www.jax.org/strain/006410; RRID:IMSR_JAX:006410) (Rossi et al., 2011). SACs are the only cholinergic neurons in retina. Pcp2-cre has restricted activity in cerebellar Purkinje neurons (https:// www.jax.org/strain/010536; RRID:IMSR_JAX:010536).

Mice were maintained on a C57/B6J or mixed C57/B6-FVB background. All animal experiments were performed in accordance with Canadian Council on Animal Care Guidelines for Use of Animals in Research and Laboratory Animal Care under protocols approved by the Centre for Phenogenomics Animal Care Committee (Toronto, Canada) and National Institutes of Health guidelines and protocols approved by Institutional Animal Use and Care Committee at Harvard University.

Histology. Anesthetized mice were either killed by decapitation and enucleated immediately or transcardially perfused with Ringer's solution followed by $4 \%$ paraformaldehyde (PFA) in PBS. Eye cups were dissected on ice in PBS and retinas fixed in $4 \%$ PFA at $4^{\circ} \mathrm{C}(6-12 \mathrm{~h}$ for nuclear markers, or $2 \mathrm{~h}$ for antibodies to GFP or ChAT). Brains were dissected and fixed in $4 \%$ PFA at $4^{\circ} \mathrm{C}$ overnight. 
Whole-mount preparations and cryosections of retinas were prepared as described previously (Lefebvre et al., 2008; Lefebvre et al., 2012). Briefly, retinas were cryoprotected in $30 \%$ sucrose-PBS, frozen, and sectioned at $20 \mu \mathrm{m}$ in a cryostat. Slides were incubated successively with blocking solution $(0.35 \%$ Triton X-100, 5\% normal donkey serum in PBS) and primary antibodies $\left(12-16 \mathrm{~h}\right.$ at $\left.4^{\circ} \mathrm{C}\right)$. Whole retinas and $75 \mu \mathrm{m}$ sections of cerebellum were incubated successively with blocking solution and then primary antibodies for $2 \mathrm{~d}$ at $4^{\circ} \mathrm{C}$. After washing, retinas and brain sections were incubated for $3 \mathrm{~h}$ at room temperature with Alexa Fluor-conjugated secondary antibodies (Thermo Fisher Scientific or Jackson ImmunoResearch). Whole retinas were flattened with photoreceptor side down onto nitrocellulose filters (Millipore).

Primary antibodies used for this study were as follows: anti-GFP (1: 2000, Aves Laboratories, catalog \#GFP-1010, RRID:AB_2307313; 1:1500, Abcam, catalog \#ab13970, RRID:AB_300798); anti-choline acetyltransferase (1:250, Millipore, catalog \#AB144P, RRID:AB_2079751); antivesicular acetylcholine transporter (1:1000, Millipore, catalog \#ABN100, RRID:AB_2630394); anti-protein kinase Ca (1:1000, Sigma-Aldrich, catalog \#P4334, RRID:AB_477345); anti-Bassoon (1:200, Cell Signaling Technology, catalog \#6897S, RRID:AB_10828496); anti-RFP/DsRed (1: 1000, Rockland, catalog \#600-401-379, RRID:AB_2209751); anti-Chx10 (1: 400, Santa Cruz Biotechnology, catalog \#sc-21690, RRID:AB_2216006); anti-Sox9 (1:500, Millipore, catalog \#AB5535, RRID:AB_2239761); antiglutamine synthetase (1:2000, BD Biosciences, catalog \#610517, RRID: AB_397879); anti-cleaved Caspase-3 (1:500, Cell Signaling Technology, catalog \#9661, RRID:AB_2341188); anti-cleaved Caspase-9 (1:300, Cell Signaling Technology, catalog \#9509, RRID:AB_2073476); anti-Brn3a (1:100, Millipore, catalog \#MAB1585, RRID:AB_94166); anti-Pax6 (1: 2000, BioLegend, catalog \#901301, RRID:AB_2565003); anti-vGlut3 (1: 1500, Synaptic Systems, catalog \#135 203, RRID:AB_887886); antisynaptotagminII (1:300, DSHB, catalog \#znp-1, RRID:AB_2315626); anti-PSD95 (1:1000, Thermo Fisher Scientific, catalog \#MA1-046, RRID: AB_2092361); anti-RPBMS (1:800, PhosphoSolutions, catalog \#1832RBPMS, RRID:AB_2492226); anti-melanopsin (1:5000, Advanced Targeting Systems, catalog \#AB-N38, RRID:AB_1608077); anti-cone arrestin (1: 1000 , C. Craft). Nuclei were labeled with DAPI, and cones were labeled with Alexa Fluor-647-conjugated peanut agglutinin (1:2000, Thermo Fisher Scientific, catalog \#L21409, RRID:AB_2315178).

In situ hybridization of retinal sections was performed as described previously (Lefebvre et al., 2008). Antisense and sense control riboprobes were synthesized using plasmid DNA templates containing cDNA sequences for the Pcdha constant region and $3^{\prime}$-UTR (Katori et al., 2009), Pcdhg constant region and $3^{\prime}$-UTR (Wang et al., 2002), and a highly conserved region of the $P c d h-b 2-b 22$ genes (Hirano et al., 2012).

Western blots. Brains or retina from postnatal day 21 (P21) to P40 and P6 mice, respectively, were dissected in ice-cold Tris-buffered saline and then homogenized in a lysis buffer containing the following: denaturing buffer (100 mm Tris-HCl, $10 \mathrm{~mm}$ magnesium acetate, $6 \mathrm{~m}$ urea, 2\% SDS, w/v), EDTA-free protease inhibitors (Roche), phosphatase inhibitor cocktails I and II (Millipore), and benzonase nuclease (10 KU, Novagen). Lysates were centrifuged at 12,000 rpm for $15 \mathrm{~min}$ and protein concentrations were determined by BCA assay (Pierce). Equal amounts of protein $(7.5 \mu \mathrm{g}$ for retinal lysates and $15 \mu \mathrm{g}$ for brain lysates) were run on a $10 \%$ SDS-PAGE gel and transferred to a polyvinylidene difluoride membrane (Millipore) overnight. Membrane were blocked for $1 \mathrm{~h}$ at room temperature in 1:1 1 $\times$ PBS and Li-Cor Odyssey Blocking Buffer and then incubated in primary antibody overnight at $4^{\circ} \mathrm{C}$. The primary antibodies used for Western blot analyses were as follows: $\alpha$-Protocadherin (1:500, Synaptic Systems, catalog \#190 003, RRID:AB_2100951); $\gamma$-Protocadherin (1: 750, Synaptic Systems, catalog \#190 103, RRID:AB_2100954); RFP/ DsRed2 (1:1000, Rockland, catalog \#600-401-379, RRID:AB_2209751); $\beta$-actin (1:10,000, Cell Signaling Technology, catalog \#3700, RRID: AB_2242334); and GAPDH (1:10,000, Cell Signaling Technology, cata$\log \# 2118$, RRID:AB_561053). Membranes were washed and incubated in appropriate secondary IRDye antibodies (1:10,000, LI-COR Biosciences, catalog \#926-32211, RRID:AB_621843; and, catalog \#926-68070, RRID:AB_10956588) and visualized on a FC Odyssey Imaging System.

$R T-P C R$ of dissociated retina cells. We used FACS to isolate live cells from dissociated P5-P7 whole retinas of transgenic mice, including
$\mathrm{OFP}^{+}$;Thy1.2 $2^{-}$SAC cells and Thy1.2 ${ }^{+}$; DRD4-GFP ${ }^{+}$RGCs, as described previously (Kay et al., 2012; Lefebvre et al., 2012). Using retinas from Thyl-OFP mice, $\mathrm{OFP}^{+}$SACs were sorted from $\mathrm{OFP}^{+}$RGCs by negative selection of Thy1.2-PE-Cy7 (Southern) labeled RGCs. Using retinas from DRD4-GFP ${ }^{+}$mice, a population of bistratified RGCs was sorted for GFP and Thy1.2-PE-Cy7. In each condition, 2000 cells were sorted directly into RNA lysis buffer (Qiagen). RNA was purified and first-strand cDNAs were generated with Superscript RT III (Invitrogen). $P c d h a$ isoforms were amplified using primers that uniquely detect the 14 Pcdha variable exon-constant exon spliced transcripts (Esumi et al., 2005). The PCR program used was as follows: $94^{\circ} \mathrm{C}$ for $2 \mathrm{~min} ; 30$ cycles of $94^{\circ} \mathrm{C}$ for $20 \mathrm{~s}, 56^{\circ} \mathrm{C}$ for $30 \mathrm{~s}, 72^{\circ} \mathrm{C}$ for $1 \mathrm{~min} ; 72^{\circ} \mathrm{C}$ for $7 \mathrm{~min}$.

Labeling of neurons. To inject recombinant adeno-associated virus (AAV) into eyes, adult mice were anesthetized with isoflurane or by intraperitoneal injection of ketamine/xylazine and a $30-1 / 2 \mathrm{G}$ needle was used to make a small hole in the temporal eye below the cornea. Then, 1.0 $\mu \mathrm{l}$ of rAAV was injected into the subretinal space with a Hamilton syringe and $33 \mathrm{G}$ blunt-ended needle, as described previously (Lefebvre et al., 2012). For cerebellar virus infection, rAAV was injected into the lateral cortical ventricle of neonates (intracerebroventricular injection), which results in the circulation of viral vectors via the CSF and transduction of neurons throughout the brain, including Purkinje cells (Passini and Wolfe, 2001). P0-P1 mice were anesthetized on ice and $1.0 \mu \mathrm{l}$ of viral vectors $(\sim 2 \times 10 \mathrm{e} 8$ genome copies diluted in $0.025 \%$ Fast Green in sterile PBS) were delivered in the right lateral cortical ventricle, as described previously (Lefebvre et al., 2012). Animals were killed and retinas were dissected 3-5 weeks after injection. The following recombinant vectors were used to label single SACs: AAV2/9.hEF1a.lox.TagBFP.lox. eYFP.lox.WPRE.hGH-InvBYF (Penn Vector Core, AV-9-PV2453) (Cai et al., 2013) and AAV2/2-CAG-memb-mCherry (Harvard Gene Therapy Institute). Individual Purkinje cells were transfected with: AAV8/DJCMV-eGFP (Vector Biolabs, 7116). AAV2/9.CAG.Flex.tdTomato. WPRE.bGH (Penn Vector Core, Allen Institute 864), AAV2/9.hSyn.HI. eGFP-Cre.WPRE.SV40 (Penn Vector Core, AV-9-PV1848). AAV.CAG. Flex.tdTomato and AAV.hSyn.HI.eGFP-Cre were coinjected to transfect and label Purkinje cells in $P c d h-a g^{\mathrm{f} / \mathrm{f}}$ pups with Cre.

Image analysis. Immunofluorescence samples were imaged using a Leica SP8 confocal microscope equipped with 405, 488, 568, and $647 \mathrm{~nm}$ lasers. For best reproduction and clarity of SAC arborizations, maximized projections of confocal images were inverted and contrast enhanced using Photoshop (Adobe Systems) or Fiji (http://imagej.net/Fiji/ Downloads; RRID:SCR_002285). Modest nonlinear enhancement was applied to some representative retina images.

Measurements of retinal layer thickness and cell number were performed as described previously (Lefebvre et al., 2008). Briefly, areas of $290 \times 290 \mu \mathrm{m}$ were chosen at equivalent retinal eccentricities from the optic nerve head. Layer thickness was measured on single optical sections adjacent to the optic nerve head. Four to six areas were measured from each retina. For quantifications of ChAT-labeled SACs, four regions $(290 \times 290 \mu \mathrm{m})$ in whole-mount retina preparations were imaged. SACs residing in the INL and GCL were quantified and combined because the two populations could not be distinguished in Pcdh-ag rko retinas. Apoptotic cells were counted on sections spanning the optic nerve head. Cells were classified as apoptotic if immunoreactive for cleaved caspase- 3 or caspase-9.

Purkinje cell dendrite self-crossings detected in single confocal planes were counted in a $70 \times 70 \mu \mathrm{m}$ region of interest located in the middle of the arbor, as described previously (Lefebvre et al., 2012). Purkinje cells from lobules 4-8 were sampled at Nyquist rates with confocal microscopy with a $0.25 \mu \mathrm{M} z$-step size with a $40 \times 1.4$ numerical aperture (NA) objective (Nikon), or $0.30-0.33 \mu \mathrm{M} Z$-step size and $76 \mathrm{~nm} X-Y$ pixel size with a $63 \times 1.3 \mathrm{NA}$ glycerol objective (Leica). A "self-crossing" was defined when two intersecting branches were detected in the same confocal plane and both dendrites were directly apposed and contained within a maximum $z$-distance of $2.0 \mu \mathrm{M}$, which is the combined diameter of two dendrites based on an average branch diameter of $\sim 0.9 \mu \mathrm{M}$ imaged with our acquisition parameters. When viewed in the orthogonal plane, the cross-sections of self-crossing dendrites could not be resolved and were displayed as continuous fluorescent structures. Overlapping branches 
that were separated by several planes could be resolved as discrete crosssections in the orthogonal plane and therefore did not meet the selfcrossing criteria. The analyst was blinded to the genotypes. Self-crossing data for each genotype were compared with data from WT sibling controls and expressed as percentage of control. Purkinje arbor areas were measured using the convex-hull selection in Fiji. Arbor thickness and planarity were analyzed using the $3 \mathrm{D}$ volume rendering and depth coding features in the LasX 3D visualization module (Leica Microsystems); confocal images were acquired with identical $z$-step number and size for equivalent depth scales. WT sibling controls were used for $P c d h a^{\text {ko }}$ and Pcdh-ag ${ }^{\text {PCko }}$ analysis. Data for Pcdhg ${ }^{\text {PCko }}\left(P c d h g^{\text {f/f }} ;\right.$ Pcp2-cre and controls Pcdhg ${ }^{+/ f}$; Pcp2-cre or Pcdhg ${ }^{+/+}$; Pcp2-cre) have been described previously (Lefebvre et al., 2012).

To calculate radial variance, maximum projections of SAC images were converted to a binary threshold and analyzed using the ImageJ plugin "Azimuthal Average" with a bin size of 8. Azimuthal Average calculates the average pixel intensity within a bin. SD between bins was calculated and then normalized against average pixel intensity of the entire SAC arbor to produce a unitless radial asymmetry index. Batch calculations for 17-19 neurons of each genotype were performed using Python3 script. Azimuthal Average also produces SAC radius measurements from soma edge to most distal branch point.

Experimental design and statistical analysis. Quantifications of cell number and neuronal morphology were performed on at least three mutant mice from multiple litters, with WT (Cre-positive and Cre-negative) or heterozygous littermates (Cre-negative) serving as controls. Animals of either sex were analyzed. The analyst was blinded to the genotypes until quantifications were complete. Statistical analyses were performed using GraphPad Prism software or Python-based statistical functions available at SciPy.org (https://docs.scipy.org/doc/scipy/reference/stats.html). All data are presented as means with SEMs unless otherwise stated. Means of two groups were compared using the two-tailed Student's $t$ test on condition of equivalent variances determined by the ANOVA $F$ test, or with the Mann-Whitney nonparametric test. Means of multiple samples were compared using one-way ANOVA and Tukey's multiple-comparisons test for pairwise analyses. Exact $p$-values are reported unless the values are $<0.0001$.

\section{Results \\ Differential and overlapping expression of $P c d h-\alpha, P c d h-\beta$, and $P c d h-\gamma$ clusters in retina}

We reported previously that $P c d h g s$ are expressed by all retinal cell types: photoreceptors in the ONL; interneurons (horizontal, bipolar, and amacrine cells) and Müller glia in the INL; and amacrine and RGCs in the GCL (Lefebvre et al., 2008). To begin this study, we assessed expression of Pcdhas and Pcdhbs in retina and compared their expression patterns with that of Pcdhgs. We designed in situ hybridization riboprobes targeting the constant exons shared by all $P c d h a$ or $P c d h g$ isoforms or a highly conserved sequence present in the majority of $P c d h b$ isoforms (Fig. $1 A$ ) (Hirano et al., 2012). Pcdha, Pcdhb, and Pcdhg mRNAs were all expressed broadly in the INL and GCL at P6 (Fig. 1B), a time at which many neurons die and dendritic self-avoidance fails in Pcdhg mutants (Lefebvre et al., 2008; Lefebvre et al., 2012). However, Pcdha mRNA was relatively more abundant in the GCL than in the INL compared with $P c d h b$ and $P c d h g$, a pattern that persisted at P14, after inner retinal development is mostly complete.

To map Pcdha expression in greater detail, we used a PcdhamCherry reporter line in which mCherry is fused to the final constant Pcdha exon within the endogenous locus, producing Pcdha proteins tagged at the carboxy terminus of the common cytoplasmic domain (Chen et al., 2017). This line is similar in design to the $P c d h g^{\mathrm{GFP}}$ line that we used previously to map Pcdhg expression (Wang et al., 2002; Lefebvre et al., 2008). The reporter confirmed the expression of Pcdhas in RGCs and in SACs in which Pcdhgs are required for dendritic self-avoidance (Fig. 1C-E). Neither of these approaches distinguished between the expression of the 12 alternate or the two C-type Pcdha variants, which diverge in sequence identity and gene regulatory mechanisms ( $\mathrm{Wu}$ and $\mathrm{Ma}-$ niatis, 1999; Wu et al., 2001; Esumi et al., 2005). We therefore profiled Pcdha isoforms by RT-PCR of RNA obtained from whole P7 retina or genetically labeled retinal subpopulations purified by FACS. Both alternate and C-type Pcdha isoforms were expressed by retinal cells, including bipolar cells, RGCs and SACs (Fig. $1 F$ and data not shown).

\section{$P c d h a$ and $P c d h b$ clusters are dispensable for retinal cell survival}

We next investigated whether Pcdhas or Pcdhbs are required for retinal development. We compared retinas from single-cluster $P c d h a$ and $P c d h b$ knock-out mice (Pcdha ${ }^{\mathrm{ko}}, P c d h b^{\mathrm{ko}}$; Chen et al., 2017), which are viable and fertile, with retinas in which $P c d h g s$ were conditionally deleted with a pan-retinal $\mathrm{Cre}\left(P c d h g^{\mathrm{ff}} ;\right.$; Six 3-cre, referred to as $P c d h g^{\text {rko }}$ for retina-specific knock-out). We detected no abnormalities in retinal architecture in $P c d h a^{\mathrm{ko}}$ and $P c d h b^{\mathrm{ko}}$ lines and the density of synapses in the plexiform layers appeared normal as judged by staining with antibodies to Bassoon, a presynaptic marker associated with neurotransmitter release sites in nerve terminals (Fig. 2A). Moreover, in contrast to thinning of the inner retinal layers in $P c d h g^{\text {rko }}$, the thickness of the INL and the IPL in $P c d h a^{\mathrm{ko}}$ and $P c d h b^{\mathrm{ko}}$ mutant retinas were similar to those in controls (Fig. 2A; quantification for Pcdha ${ }^{\text {ko }}$ in Fig. $2 J$ ). We used Western blotting to determine whether upregulation of Pcdhgs could compensate for the loss of Pcdhas, but found no change in Pcdhg protein levels in $P c d h a^{\mathrm{ko}}$ mutant retinas (Fig. 2B). Therefore, in contrast to Pcdhg, the Pcdha and Pcdhb gene clusters are dispensable for major aspects of retinal development, including neuronal generation and survival.

\section{Inactivation of both the Pcdha and Pcdhg clusters results in a synergistic loss of inner retinal layers}

$P c d h a, P c d h b$, and $P c d h g$ genes are expressed in overlapping populations of retinal cells and the proteins that they encode are highly homologous. Therefore, although Pcdha or Pcdhb deletion alone did not have a striking retinal phenotype, functional compensation by the Pcdhgs might mask their roles. To test this possibility, we set out to generate a mouse mutant lacking multiple Pcdh clusters. Deletion of the three Pcdh clusters is complicated by the locus size (1 MB; Wu and Maniatis, 1999) and the presence of the Taf7 gene, which encodes a component of the transcription machinery, between the Pcdhb and Pcdhg clusters. Taf7 mutants die between embryonic days 3.5 and 5.5 (Gegonne et al., 2012; Hasegawa et al., 2016). Accordingly, triple cluster Pcdh mutant mice require transgenic restoration of the Taf 7 gene to develop to term (Hasegawa et al., 2016; Mountoufaris et al., 2017). Even with Taf7 restored, triple cluster mice die soon after birth, likely due to the neonatal lethality caused by constitutive deletion of Pcdhg (Wang et al., 2002). To circumvent this limitation, we used the Crispr-Cas9 system to mutate Pcdha in the conditional $P c d h g^{\mathrm{f}}$ floxed allele (Lefebvre et al., 2008), choosing Pcdha over Pcdhb, because it has been studied in greater detail (Lefebvre, 2017). We used an sgRNA designed to target the Pcdha constant exon 1 because, in our previous studies, targeting of a constant exon in the Pcdhg locus generated a null allele (Wang et al., 2002; Lefebvre et al., 2008; Prasad et al., 2008). The sgRNA and Cas9 were injected into $P c d h g^{\mathrm{f}}$ zygotes to generate $P c d h a^{\text {conexldel }} ; P c d h g^{\mathrm{f}}$ mice, hereafter called $P c d h$ - $a g^{\mathrm{f}}$ (Fig. 2C).

We chose three $P c d h-a g^{\mathrm{f}}$ founders with deletions of $>100 \mathrm{bp}$ spanning the exon-intron site that are predicted to disrupt splic- 
A

(14 isoforms)

Pcdh-beta

Pcdh-gamma

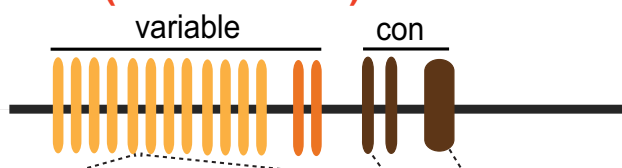

(22 isoforms)

(22 isoforms) constant

variable exons

(con)

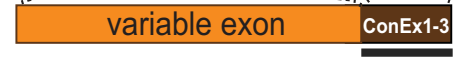

EC1 EC2 EC3 EC4 EC5 EC6 TM ICR

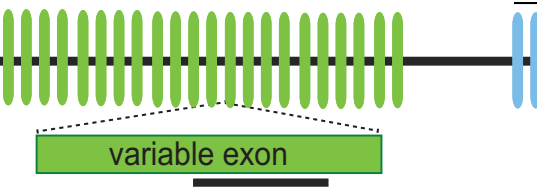

EC1 EC2 EC3 EC4 EC5 EC6 TM 웅

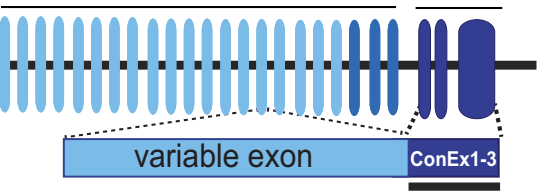

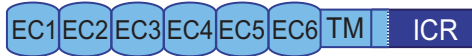
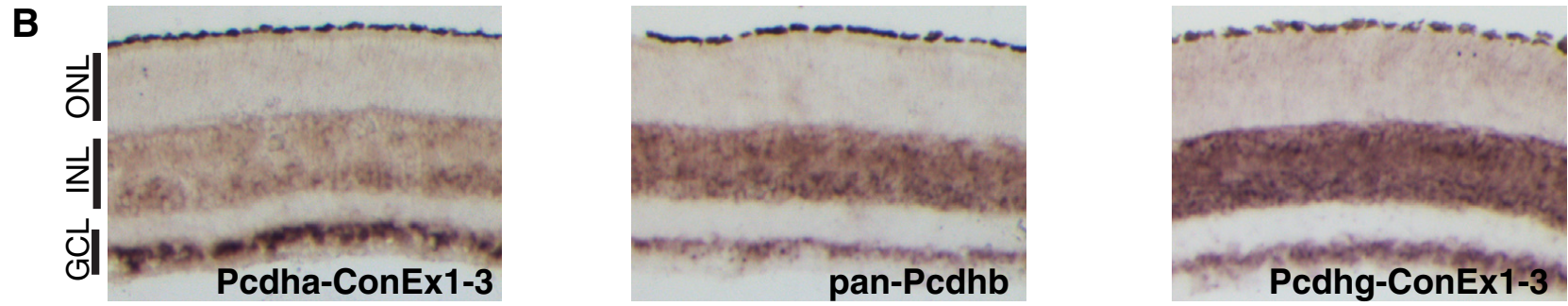

C

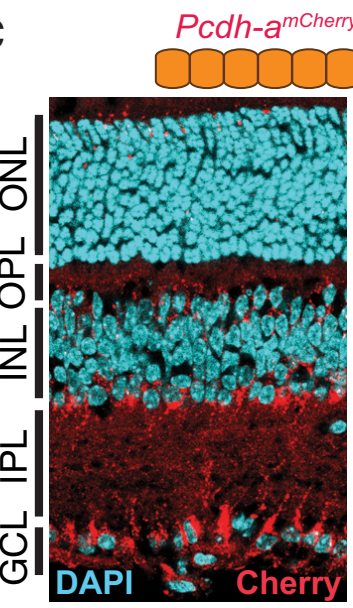

knock-in

1) Cherry

D
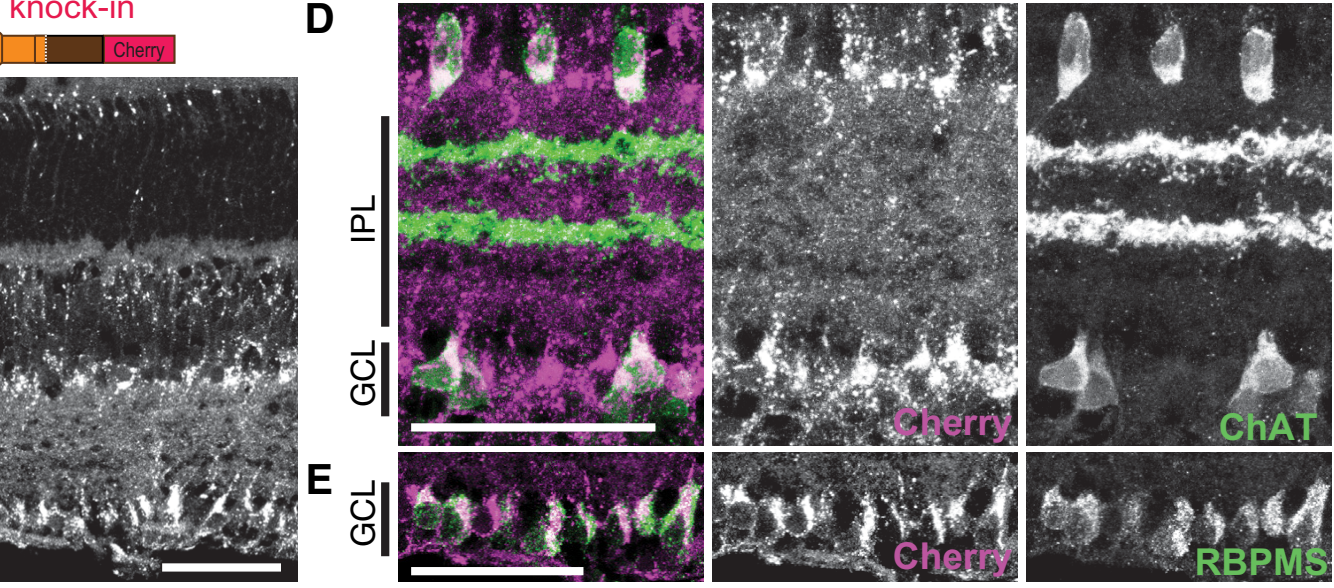

$\mathbf{F}$

Pcdha1 a2 a3 a4 a5 a6 a7 a8 a9 a10 a11a12 c1 c2 PgC3
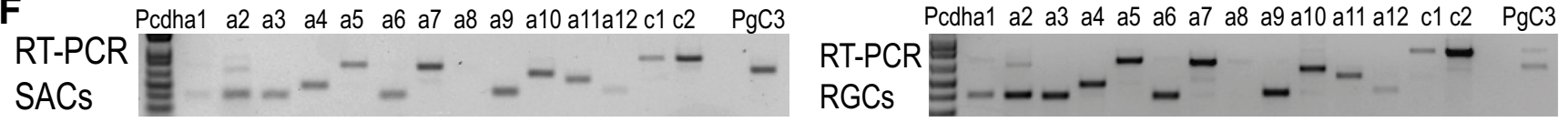

Figure 1. Expression of the clustered protocadherins in mouse retina. $A$, Schematic representation of the $P c d h$ locus comprising the $P c d h a, P c d h b$, and $P c d h g$ clusters. $P c d h a$ and $P c d h g$ mRNAs are assembled by splicing a variable exon to three constant exons (ConEx 1-3). Each variable exon is transcribed from its own promoter. Black bars represent region targeted by in situ hybridization riboprobes. Bottom, Pcdha, Pcdhb, and Pcdhg isoforms share similar protein organization, including six extracellular calcium motifs (EC), one transmembrane domain, and an intracellular region (ICR). $\boldsymbol{B}$, In situ hybridization of P7 retinas showing Pcdha, Pcdhb, and Pcdhy mRNA expression in the INL and the GCL. C-E, Pcdha expression revealed by a Pcdha-mCherry fusion protein (C, top) generated by insertion of $m$ Cherry at the $3^{\prime}$ end of the $P c d h a$ coding region in the endogenous locus. Sections from P14 retinas show expression of Pcdha-mCherry proteins in the INL and, $G C L$ ( $C$, red, with DAPI in cyan). Costaining with antibodies to mCherry confirm expression of Pcdhas in SACs ( $\boldsymbol{D}$, ChAT, green), and RGCS ( $\boldsymbol{E}$, RBPMS, green). $\boldsymbol{F}$, RT-PCR of alternate (a1-a12) and C-type (C1, C2) Pcdha transcripts from RNA extracts of SACs and a subpopulation of RGCs purified by FACS. Scale bars, $50 \mu \mathrm{m}$.

ing and alter the protein coding sequence for the Pcdha intracellular domain (Fig. 2C). Using an antibody targeting the constant region, we confirmed the absence of full-length Pcdha proteins in $P c d h-a g^{\mathrm{f} / \mathrm{f}}$ brain lysates (Fig. $2 D$ ). Due to lack of available antibodies that recognize Pcdha ectodomains, we could not test for the presence of truncated Pcdha proteins. Therefore, we investigated whether our mutants displayed a previously documented effect of Pcdha deletion on the clumping and disorganization of serotonergic axons in the hippocampus (Katori et al., 2009; Chen et al., 2017). Defects in serotonergic projections were similar in $P c d h-a g^{\mathrm{f} / \mathrm{f}}$ homozygotes, $P c d h a^{\mathrm{ko} / \mathrm{ko}}$ homozygotes, and $P c d h a^{\mathrm{ko}}$; Pcdh-ag transheterozygotes (data not shown), confirming that the Crispr-mediated deletions effectively disrupted Pcdha func- tion. In subsequent experiments, we used two lines, $P c d h-a g^{\mathrm{f}}-1$ and $P c d h-a g^{\mathrm{f}}-3$, interchangeably; results from the two were indistinguishable.

We expected that $P c d h-a g^{\mathrm{f} / \mathrm{f}}$ mice would be equivalent to $P c d h a^{\text {ko/ko }}$ in the absence of Cre and therefore viable. We were therefore surprised to recover few $P c d h-a g^{\mathrm{f} / \mathrm{f}}$ mutant animals from crosses between $P c d h-a g^{\mathrm{f} /+}$ mice. Moreover, compared with WT littermates, $P c d h-a g^{\mathrm{f} / \mathrm{f}}$ mice were smaller and exhibited a hunched posture, stiffness, and limb tremors (Fig. 2E). These phenotypes resemble those of $P c d h g^{\text {ko }}$ neonates and are thought to result from an excessive neuronal loss in the spinal cord and brainstem (Wang et al., 2002; Hasegawa et al., 2017). Surviving $P c d h$-ag animals were also smaller at the time of weaning (Fig. 2F; 

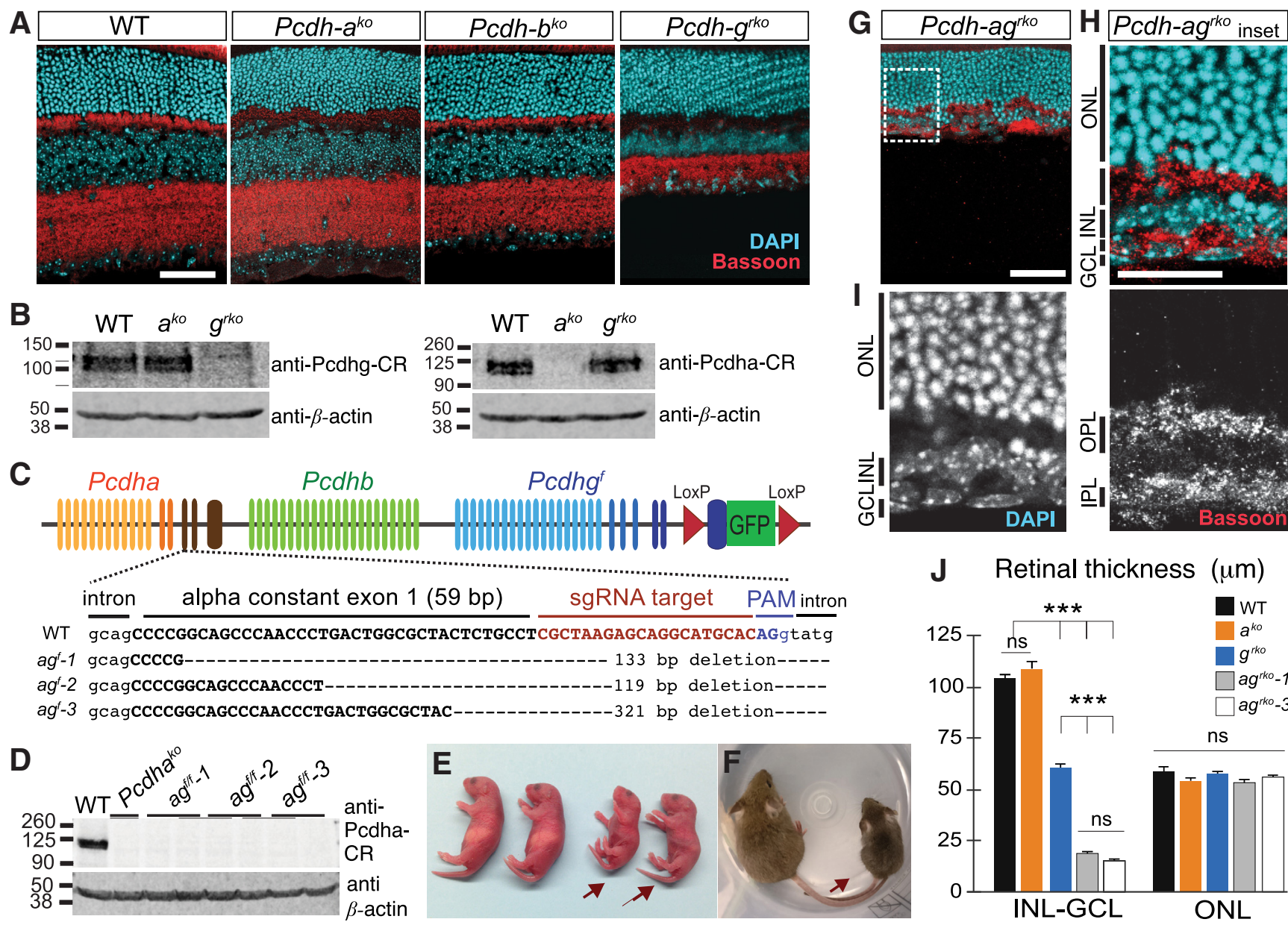

Figure 2. Synergistic loss of inner retinal structures in $P c d h$-ag double mutant retinas. $A$, Retina sections from WT, $P\left(d h a^{\mathrm{ko}}, P c d h b^{\mathrm{ko}}\right.$, and $P c d h g^{\text {rko }}$ animals labeled with anti-Bassoon (red) to mark synapses and DAPI (cyan) to mark cells. B. Western blots of $P$ cdha and $P c d h$ proteins in $P c d h a^{\mathrm{ko}}$ and $P c d h g^{\mathrm{rko}}$ retinas using antibodies to the constant region (CR) shared by all isoforms in each cluster. C, CRISPR/Cas9 targeting of Pcdha constant exon 1 (capital letters) in $P c d h g^{f}$ allele. Three $P c d h-a g^{f}$ founders with deletions spanning exon-intron junction: $\mathrm{ag}^{\mathrm{f}}-1$, ag ${ }^{\mathrm{f}}-2$, and ag ${ }^{\mathrm{f}}-3$.

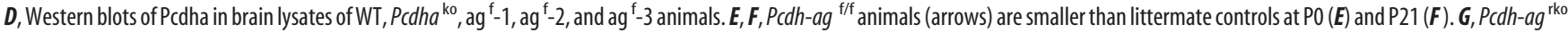
retina labeled with anti-Bassoon (red) and DAPI (cyan). $\boldsymbol{H}, \boldsymbol{I}$, Higher magnification of boxed region in G.J, Comparisons of inner retina (INL, IPL, and GCL combined) and outer retina (ONL) thickness in single and double $P c d h$ mutant retinas. $P\left(d h-a g^{\text {rko }}\right.$ retinas from ag ${ }^{f}-1$ and ag $^{f}-3$ lines have similar reductions (ns, not significant, $p=0.62$, post hoc Tukey test). Data show mean \pm SEM. $n=$ 18 sections from $3-5$ animals per genotype. INL: one-way ANOVA, $F_{(4,85)}=6.4, p<0.0001$; pairwise Tukey's multiple-comparisons tests, ${ }^{* * *} p<0.0001$. ONL: one-way ANOVA, $F_{(4,85)}=2.4, p=$ 0.071; pairwise Tukey's multiple-comparisons tests, not significant (ns). Scale bars: $\boldsymbol{A}, \mathbf{G}, 50 \mu \mathrm{m} ; \boldsymbol{H}, 25 \mu \mathrm{m}$.

controls $=12.0 \pm 0.5 \mathrm{~g} ;$ Pcdh $-a \mathrm{~g}^{\mathrm{f} / \mathrm{f}}=6.9 \pm 0.6 \mathrm{~g}, n=13$ animals, $t_{(24)}=8.0, p<0.0001$, unpaired $t$ test, $)$. The likely explanation is that the targeted Pcdhg ${ }^{\mathrm{f}}$ allele, even in the absence of Cre, is hypomorphic (Lefebvre et al., 2008; Prasad et al., 2008). Therefore, although $P c d h g^{\mathrm{f} / \mathrm{f}}$ animals are viable and fertile, decreased levels of Pcdhgs in Pcdh-ag ${ }^{\mathrm{f}}$ would lead to decreased viability. This result provided an initial indication that postnatal viability is affected by interactions between Pcdhas and Pcdhgs.

To seek retinal phenotypes, we crossed $P c d h$-ag ${ }^{\mathrm{f}}$ mice to a Six3-cre line that is expressed in retinal progenitors to limit $P c d h g$ deletion to retina and analyzed survivors $\left(P c d h-a g^{\mathrm{f} / \mathrm{f}}\right.$; Six3-cre, referred to as $P c d h-a g^{\text {rko }}$, rko indicating pan-retinal and retinaspecific knock-out). We avoided use of Pcdh-ag $\mathrm{g}^{\mathrm{fff}}$ mice as controls, instead using Cre-positive or Cre-negative WT mice or Crenegative $P c d h g^{\mathrm{f} /+}$ littermates. At P21, when retinal architecture is fully developed in controls, the inner retina was nearly eliminated in $P c d h$ - $a g^{\text {rko }}$ mice. All retinal layers were present, but the INL and IPL were dramatically thinned (Fig. $2 G$ ). The INL contained only a few layers of cells, whereas the IPL was reduced to a thin layer of processes (Fig. $2 \mathrm{H}, \mathrm{I}$ ). Remarkably, the ONL comprising the photoreceptors remained intact. We measured the combined thickness of the inner retinal layers (INL, IPL, and GCL). Compared with the inner retinal layers in $P c d h{ }^{\mathrm{ko}}$ retinas (unchanged, $108.8 \pm 3.3 \mu \mathrm{m}$ compared with WT control $103.9 \pm 1.9 \mu \mathrm{m}, p=$ 0.35 , Tukey's pairwise comparison) and in $P c d h{ }^{\text {rko }}$ retinas (reduced by $40 \%, 60.9 \pm 1.4 \mu \mathrm{m}$ ), the layers were reduced by $>80 \%$ in double $P c d h-a g^{\text {rko }}$ mutant retinas $\left(a g^{\text {rko }}\right.$ line $1: 18.6 \pm 0.6 \mu \mathrm{m}$; $a g^{\text {rko }}$ line 3: $15.4 \pm 0.5 \mu \mathrm{m} ; n=18$ sections, from $3-5$ animals per genotype, one-way ANOVA $F_{(4,85)}=6.4, p<0.0001$; Tukey's multiple-comparisons test between $a g^{\text {rko }}$ lines and single $P c d h$ mutants, $p<0.0001$; Fig. $2 J$ ). In contrast, the thickness of the OPL was unaffected. Therefore, Pcdhas and Pcdhgs act synergistically to form or maintain the inner retina.

\section{Developmental cell death of inner retinal neurons in}

Pcdh-ag ${ }^{\text {rko }}$ mutant retinas

We next investigated whether retina thinning in $P c d h-a g^{\text {rko }}$ mutants results from reduced numbers of inner retinal neurons. Numbers of bipolar cells (Chx10+), amacrine cells (Pax6+), and RGCs (Brn3a + ) were unaffected in single $P c d h a^{\mathrm{ko}}$ retina, consistent with results presented above, but reduced far more in double $P c d h-a g^{\text {rko }}$ than in single $P c d h g^{\text {rko }}$ retina: $\sim 80 \%$ and $72 \%$ reduc- 


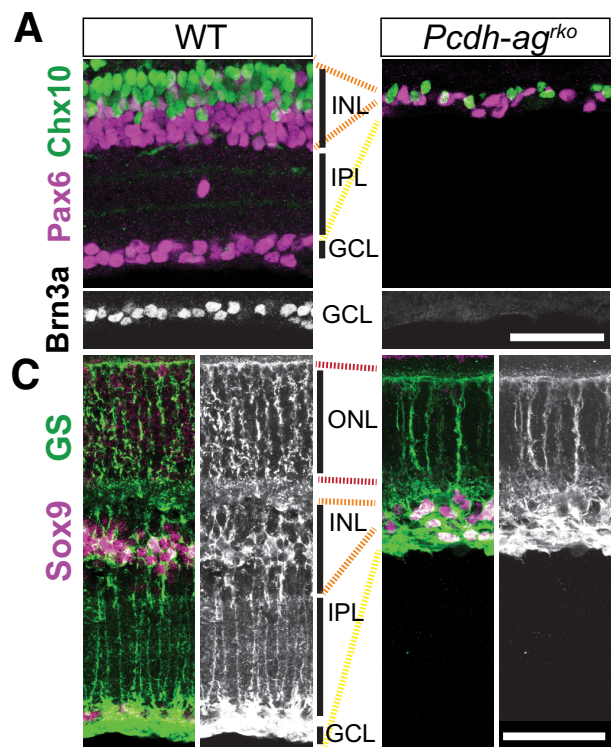

D RGC markers

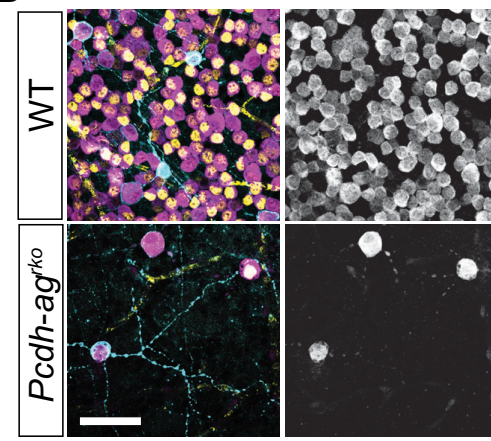

Melanopsin

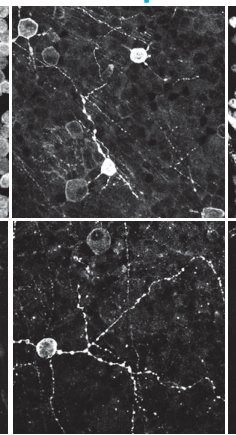

B

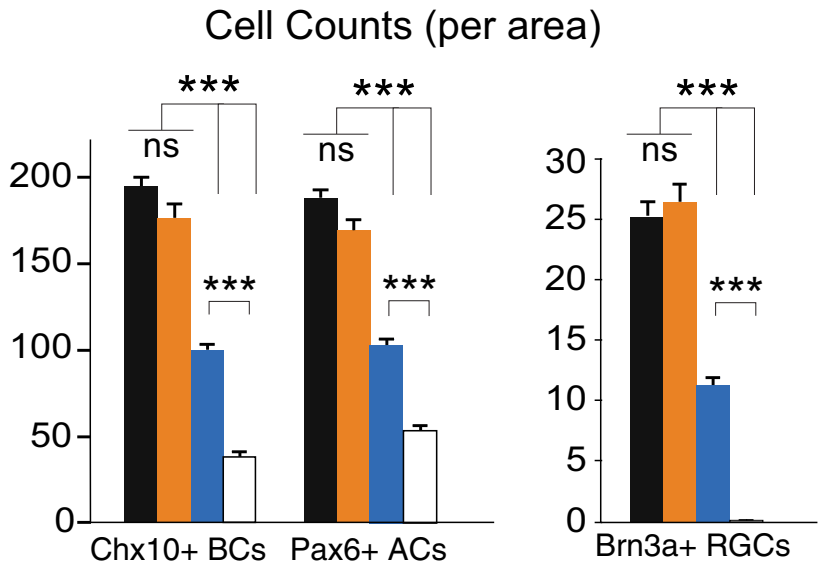

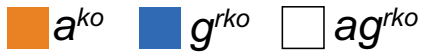

Brn3a

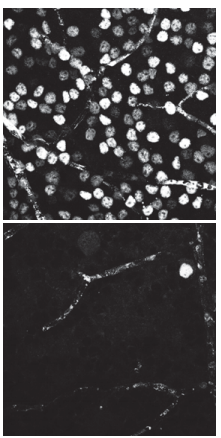

E

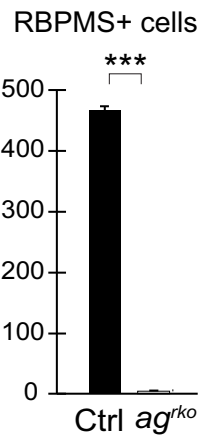

F

$\%$ Total RGC

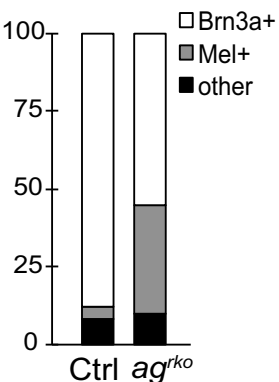

Figure 3. Synergistic loss of interneurons and RGCs in $P c d h$-ag double mutant retinas. $\boldsymbol{A}-\boldsymbol{C}$, Sections of P21 WT and $P$ cdh-ag ${ }^{\text {rko }}$ retinas immunostained for retinal cell markers. $\boldsymbol{A}$, Chx $10+$ bipolar cells (BC, green), Pax6 + amacrine cells (AC, magenta), and Brn3a + RGCs (white) in WT and Pcdh-ag ${ }^{\text {rko }}$ retinas. $B$, Quantifications of BCs, $A C s$, and RGCs in $P c d h a^{\mathrm{ko}}, P c d h{ }^{\mathrm{k} o}$, and $P\left(d h-a g^{\text {rko }}\right.$ retina sections from 4- 6 animals per genotype. Chx10 counts: one-way ANOVA, $F_{(3,76)}=217.3, p<0.0001, n=20$ sections. Tukey's multiple-comparisons tests, $p<0.0001$. Pax 6 counts: one-way ANOVA, $F_{(3,76)}=260.3, p<0.0001, n=20$ sections. Brn3a counts: one-way ANOVA, $F_{(3,60)}=165.1, p<0.0001, n=16$ sections. $C$, Müller glia immunolabeled for glutamine synthetase (green) and Sox9 (magenta). $\boldsymbol{D}-\boldsymbol{F}$, Analysis of RGC survival in Pcdh-ag ${ }^{\text {rko }}$ and WT whole-mount retinas. D, Immunostaining with RGC markers (RBPMS, magenta; melanopsin, cyan; Brn3a, yellow). $\boldsymbol{E}$, Counts of cells labeled with antibodies to RBPMS, a pan-RGC marker. $n=12$ retina sections, 3 animals per genotype, $p<0.0001$, Mann-Whitney $U$ test. $\boldsymbol{F}$, Analysis of two mutually exclusive Brn3a + and $\mathrm{Mel}+\mathrm{RGC}$ populations expressed as percentage total RGCs among surviving RGCs in Pcdh-ag ${ }^{\text {rko }}$ retinas. Data are shown as means \pm SEM. ${ }^{* * *} p<0.0001$. Scale bars, $50 \mu \mathrm{m}$.

tions for bipolars and amacrines in $P c d h-a g^{\text {rko }}$, respectively, compared with $\sim 50 \%$ and $45 \%$ in Pcdhg ${ }^{\text {rko }}$ mutants (Fig. $3 A, B$; Chx10, $n=20$ sections: one-way ANOVA, $F_{(3,76)}=217.3, p<$ 0.0001; Pax6, $n=20$ sections: one-way ANOVA, $F_{(3,76)}=260.3$, $p<0.0001$; Tukey's multiple-comparisons test, $p<0.0001$ between genotypes, except for the WT and $P c d h a^{\text {ko }}$ pair). Most dramatically, only $1 \%$ of RGCs persisted in $P c d h-a g^{\text {rko }}$ retina (one-way ANOVA, $F_{(3,60)}=165.1, p<0.0001$; Tukey's multiplecomparisons test, $p<0.0001$ between genotypes, except for the WT and $P c d h a^{\text {ko }}$ pair, $\left.p=0.85\right)$. Müller glia cells were also reduced in number but maintained processes spanning the retina (Fig. 3C). Photoreceptors were spared in both $P c d h-g^{\text {rko }}$ retinas (Fig. 2A,J; Lefebvre et al., 2008) and Pcdh-ag ${ }^{\text {rko }}$ retinas (Fig. $2 G, J)$.

We stained retinal whole mounts to characterize the few surviving RGCs in Pcdh-ag ${ }^{\text {rko }}$ mutants. Examination of the entire RGC population with a pan-RGC marker confirmed their near elimination, with a mere $1.1 \%$ of RGCs remaining in $P c d h-a g^{\text {rko }}$ retinas compared with controls $(n=12$ retina sections, 3 animals per genotype, $p<0.0001$, Mann-Whitney $U$ test; Fig. $3 D, E)$. We next quantified two mutually exclusive RGC cell types, the Brn3a+ RGCs and the melanopsin-positive intrinsically photo- sensitive RGCs (Mel+ ipRGCs) (Rodriguez et al., 2014). Both surviving RGC subpopulations are sparse, but proportionally more $\mathrm{Mel}+\mathrm{ipRGCs}$ remained in mutant $P c d h-a g^{\text {rko }}$ retinas $(4.1 \%$ of total RGCs are Mel+ in control compared with $35 \%$ of Mel +/total RGCs in Pcdh-ag ${ }^{\text {rko }}$ mutants; Fig. $\left.3 D-F\right)$. The enrichment of $\mathrm{Mel}+$ ipRGCs among surviving RGCs indicates a resistance to cell death, which has also been observed for ipRGC subpopulations in injury models such as axotomy and optic neuropathies (Pérez de Sevilla Müller et al., 2014; Cui et al., 2015; Duan et al., 2015).

We also examined retinas at earlier and later times to determine when Pcdhs act. RGCs and amacrine cells were present in $P c d h-a g^{\text {rko }}$ mutant retinas at P0 but had severely declined in number by P6 (Fig. 4A-D). RGCs were already eliminated by this time, revealing the rapid loss of RGCs within the first postnatal days (Fig. 4C,D). During this time, populations of inner retinal cells are reduced by a stereotyped wave of developmental cell death (Young, 1984). In Pcdh-ag ${ }^{\text {rko }}$ mutant retinas, more apoptotic cells were detected in mutant than in control INL at P6 by staining for cleaved-caspase 9 and cleaved-caspase 3 (Fig. 4E,F), indicating that loss of retinal cells occurs from accentuated cell death $(n=18 \mathrm{sec}$ tions, $4-5$ animals per genotype; CC3, $p=0.0051$; CC9, $p=0.0010$, Mann-Whitney test). These data support the idea documented pre- 
viously for Pcdhgs that Pcdhs promote neuronal survival rather than attenuating neurogenesis (Lefebvre et al., 2008). Defects in $P c d h-a g^{\text {rko }}$ mutant retinas were not detectably more severe at P70 than at P21, further indicating that cell loss is developmentally restricted (Fig. 4G,H). Together, these results demonstrate that Pcdha and $P c d h g$ act synergistically and redundantly to promote survival of retinal neurons during early postnatal life.

\section{Degraded sublaminar organization of plexiform layers in Pcdh-ag ${ }^{\text {rko }}$ mutant retinas}

A key feature of retinal circuit organization is that axons and dendrites of specific neuronal types are restricted to one or a few of $\sim 10$ sublaminae within the IPL and one of two sublaminae within the OPL, thus constraining their choice of synaptic partners (Sanes and Zipursky, 2010; Lefebvre et al., 2015). We showed previously that Pcdhgs are dispensable for sublamina-specific arborization of processes in the IPL and OPL (Lefebvre et al., 2008) and used similar methods to show that sublaminar organization persists in $\mathrm{Pcd}$ $h{ }^{\mathrm{ko}}$ retina (data not shown). We investigated whether this preservation reflects redundant functions of Pcdhas and Pcdhgs. In the IPL, processes of OFF and ON bipolar subtypes are segregated to distal and proximal IPL sublayers, respectively. Sections were double labeled with antibodies to Syt2, which labels Type 2 OFF cone bipolars, and to protein kinase $\mathrm{C} \alpha$, which labels $\mathrm{ON}$ rod bipolars. Axon terminals labeled by these markers were clearly segregated in control retina but were intermingled in $P c d h-a g^{\text {rko }}$ retina (Fig. 5A, B). We also labeled two amacrine cell types: SACs with antibodies to choline acetyltransferase (ChAT) and a narrowfield subtype with vGluT3. In control, the two SAC populations residing in the INL and in the GCL form two segregated dendritic layers in the IPL, whereas the vGluT3 + amacrine cells form processes between them (Fig. $5 C$ ). In $P c d h-a g^{\text {rko }}$ retinas, this segregation was lost as the narrow ChAT + bands collapsed (Fig. 5D). Moreover, nearly all vGluT3+ amacrine cells were absent.

In the OPL, synapses of rod terminals (spherules) on rod bipolar dendrites are concentrated in an outer sublamina, abutting the ONL, whereas synapses of cone terminals (pedicles) on cone bipolar dendrites occupy an inner sublamina, abutting the INL (Fig. 5E). This sublaminar organization was also disrupted in $P c d h-a g^{\text {rko }}$ retina, with rod spherules and cone pedicles intermixed (Fig. $5 F$ ). Cone photoreceptor morphology was intact in mutants (Fig. $5 F$ ) but PNA labeling revealed fragmentation at the base of cone pedicles, suggesting disorganized contacts with postsynaptic partners (Fig. 5G). Therefore, the combined loss of the Pcdha and Pcdhg clusters has devastating consequences on layers. Scale bars, $50 \mu \mathrm{m}$.
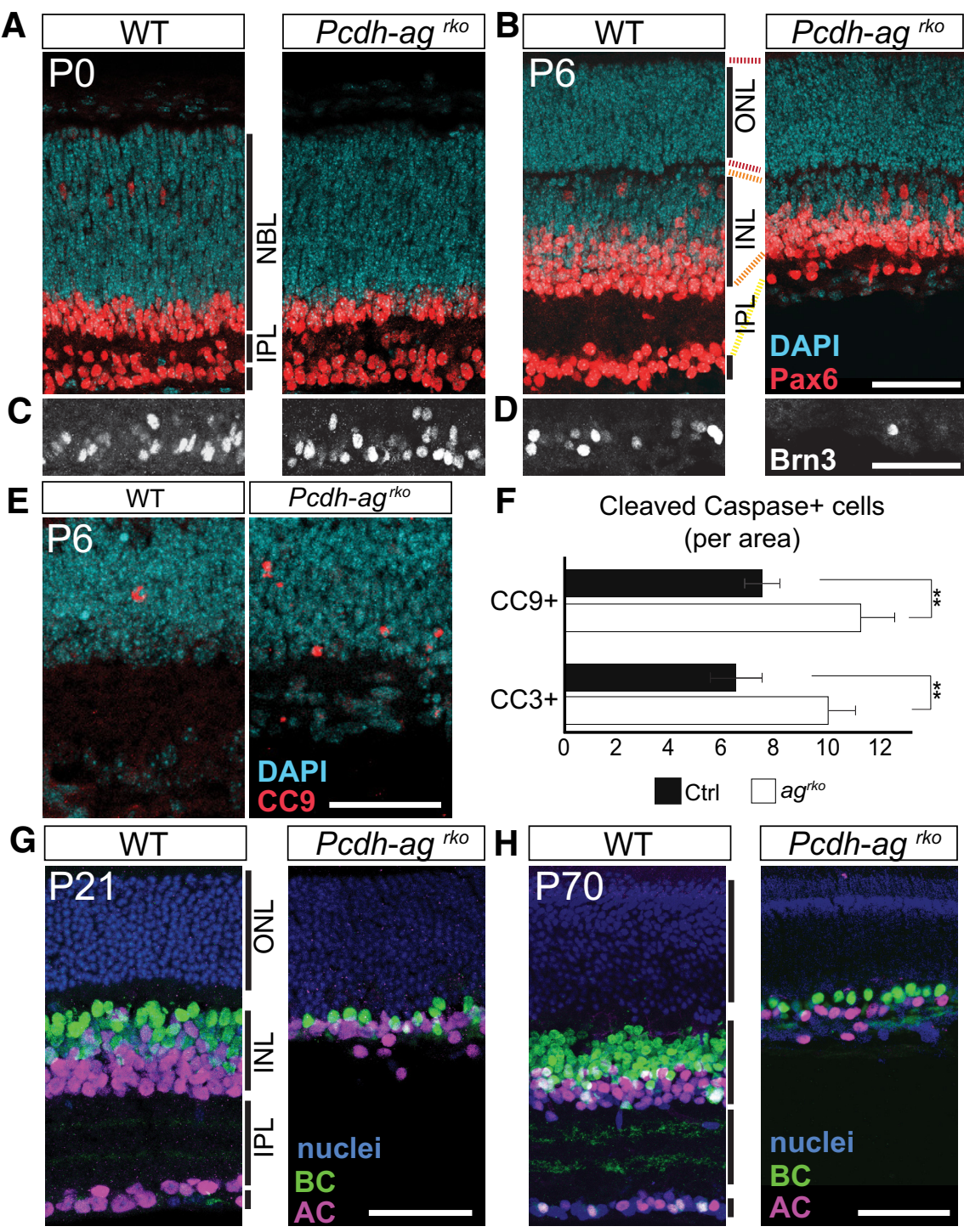

Figure 4. Retinal cell loss from accentuated postnatal cell death in double $P$ cdh-ag mutant retinas. $A-D$, Immunolabeling of $\mathrm{ACs}$ (Pax6+, red; $\boldsymbol{A}, \boldsymbol{B})$ and $\mathrm{RGCs}(\mathrm{Brn} 3 \boldsymbol{C}+$, white; $\boldsymbol{C}, \boldsymbol{D})$ reveal decline in $\mathrm{RGC}$ and $\mathrm{AC}$ populations in $P$ cdh-ag ${ }^{\text {rko }}$ retinas from $\mathrm{PO}(\boldsymbol{A}$, $\boldsymbol{C})$ to P6 (B, D) compared with WT. NBL, neuroblastic layer. $\boldsymbol{E}$, Apoptotic cells marked by cleaved caspase-9 (CC9) in control and $a{ }^{\text {rko }}$ retinas. Data show mean \pm SEM from 4 -5 animals per genotype. CC3: $p=0.0051$, Mann-Whitney U test, $n=18$ sections CC9: $p=0.001$, Mann-Whitney U test, $n=18$ sections. ${ }^{* *} p<0.01$. G, H, Comparisons of AC and BC populations in Pcdh-ag ${ }^{\text {rko }}$ green, and DAPI in blue. $\boldsymbol{H}$, Anti-AP2 is shown in magenta; anti-Chx10 in green, and T0-PRO in blue. Dashed lines indicate retinal

many aspects of IPL and OPL development. Disruptions to sublaminar organization may be in part a consequence of neuronal loss. For example, the number of bipolar cells is reduced by $\sim 80 \%$ (Fig. $3 B$ ) and vGlut $3+$ amacrine cells are almost completely eliminated in $P c d h$ - $a g^{\text {rko }}$ retina (Fig. 5D). Alternatively, Pcdhs may mediate interactions among processes required for their segregation. At present, we cannot distinguish between these possibilities.

We examined neonatal retinas to determine whether IPL lamination was disrupted from the outset or degraded during development. Little bipolar stratification occurs until the second postnatal week, but SACs form lamina-restricted arbors in neonates. Processes of SACs were laminated similarly in control and $P c d h-a g^{\text {rko }}$ retinas at P0 but had collapsed by P6 (Fig. $5 H$ ). These 

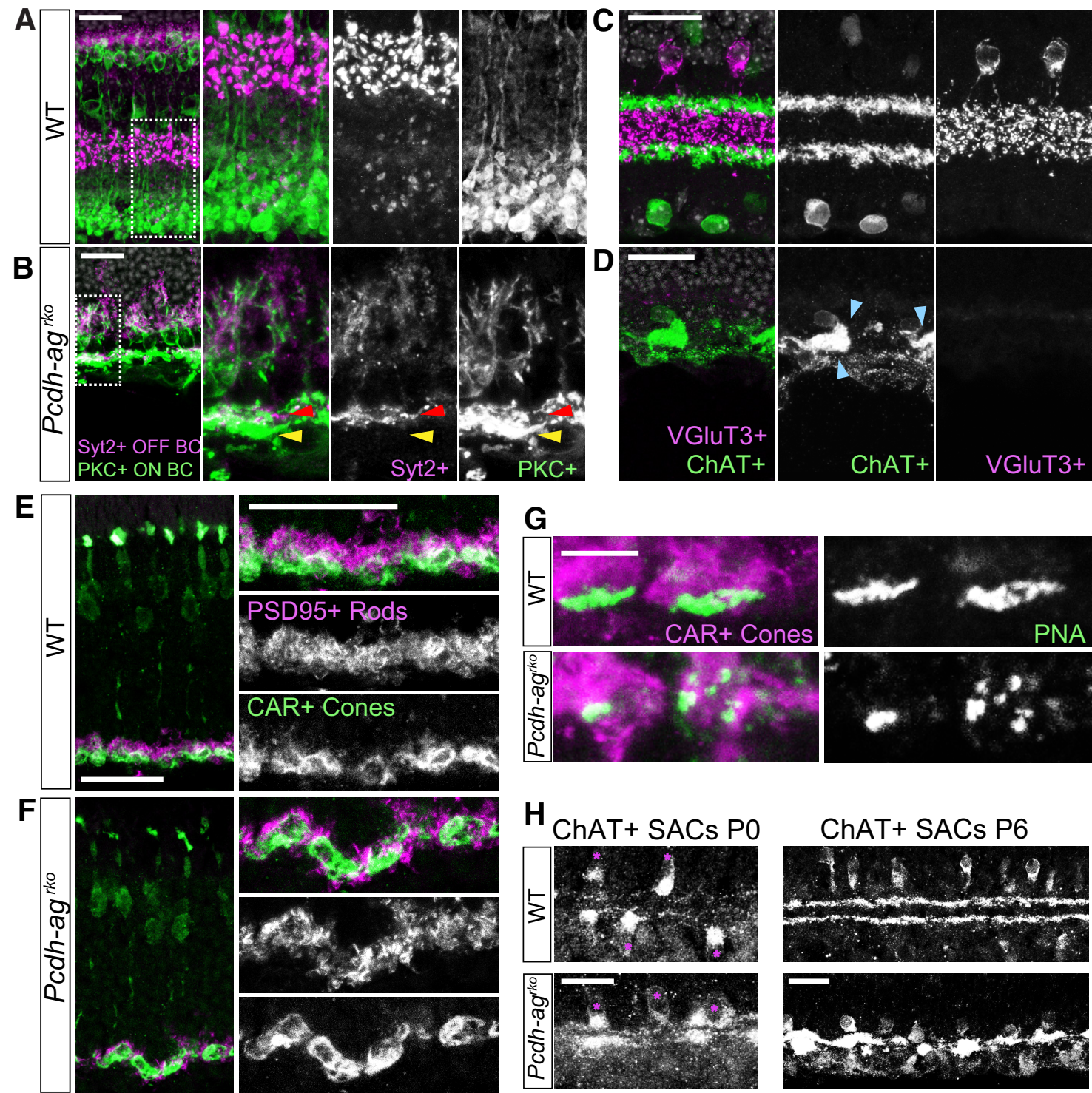

Figure 5. Laminar organization of the IPL and OPL is not maintained in the absence of $P c d$ ha and $P$ cdhg clusters. $A, B$, Sections of inner retina showing laminar segregation of OFF cone bipolar (Syt2 + , magenta) and ON rod bipolar axon terminals (PKC $\alpha+$, green) in IPL of P21 WT $(\boldsymbol{A})$ and $P c d h-a g^{\text {rko }}$ retinas $(\boldsymbol{B})$. White boxes in left panels indicate regions shown at higher magnification in the other panels. Arrows indicate OFF (red) and ON (yellow) BC terminals, which are intermingled in Pcdh-a ${ }^{\text {rko }}$. C, $\boldsymbol{D}$, Laminar segregation of AC processes: vGluT3+ narrow field ACs (magenta) form elaborate processes between two ChAT + SAClayers (green) in WT (C) at P21. In Pcdh- $a g^{\text {rko }}$ retinas (D), vGluT3 + ACs are absent and ChAT + layers are collapsed. Blue arrows denote clumping of SAC dendrites. $\boldsymbol{E}, \boldsymbol{F}$, Sections of outer retina showing laminar organization of rod (PSD95+, magenta) and cone (CAR+, green) terminals in OPL. $\boldsymbol{E}$, WT. $\boldsymbol{F}$, Rod spherules are displaced relative to cone pedicles in $P c d h-a g^{\text {rko }}$. G, Lectin PNA labeling show fragmentation of cone synaptic structures in Pcdh-ag ${ }^{\text {rko }}$ retinas. $\boldsymbol{H}$, SAC processes are stratified in WT and $P c d h-a g^{\text {rko }}$ retina at PO (left), but are collapsed in $P c d h-a g^{\text {rko }}$ retina at $P 6$ (right). Scale bars: $\boldsymbol{A}-\boldsymbol{F}, \boldsymbol{H}, 25 \mu \mathrm{m} ; \boldsymbol{G}, 5 \mu \mathrm{m}$.

data demonstrate that IPL disruption in double Pcdh mutants becomes more pronounced during postnatal development.

\section{Dose-dependent requirement for Pcdhas and Pcdhgs}

To investigate systematically the relative contributions of Pcdhas and Pcdhgs to retinal development, we analyzed Pcdh gene cluster dosage in a series of mutant retinas lacking one or both copies of the Pcdha or Pcdhg clusters. For example, we crossed Pcdh$a g^{\text {rko/+ }}$ to $P c d h-a^{\text {ko }}$ mice to generate $P c d h a^{\text {rko/ko }} ; g^{\text {rko/wt }}$ retinas (abbreviated here as $P c d h-a^{0} g^{1}$, in which $a^{0}$ denotes no WT Pcdha allele and $g^{1}$ denotes one WT Pcdhg allele) and Pcdh$a g^{\text {rko/+ }}$ to $P c d h-g^{\text {rko }}$ mice to generate $P c d h-a^{\text {rko/wt }} ; g^{\text {rko/rko }}$ retinas $\left(P c d h-a^{1} g^{0}\right)$. Overall, the series included retinas from seven genotypes with decreasing cluster dosage: four $\left(P c d h-a^{2} g^{2}\right)$, two
$\left(P c d h-a^{1} g^{1}, P c d h-a^{0} g^{2}, P c d h-a^{2} g^{0}\right)$, one $\left(P c d h-a^{1} g^{0}, P c d h-a^{0} g^{1}\right)$, or zero $\left(P c d h-a^{0} g^{0}\right) P c d h a$ or Pcdhg alleles (Fig. $\left.6 A\right)$.

To quantify the degree of cell loss in this allelic series, we measured the thickness of the inner retina (INL, IPL, and GCL combined) and the numbers of bipolar, amacrine, and RGCs, expressing each as a percentage of the value quantified in littermates. Cell loss was exquisitely sensitive to $P c d h$ dosage, revealing cluster-specific and dose-dependent survival activities of Pcdhs (Fig. 6A-E). When both copies of the Pcdha cluster were deleted, a single WT copy of $P c d h g$ was insufficient to support survival: the numbers of bipolar cells and RGCs were significantly reduced in $P c d h-a^{0} g^{1}$ retinas compared with control retinas (by $18 \%$ and $32 \%$, respectively; $p<0.0001$, post hoc Tukey pairwise test). However, a single copy of the Pcdha cluster was able to compen- 


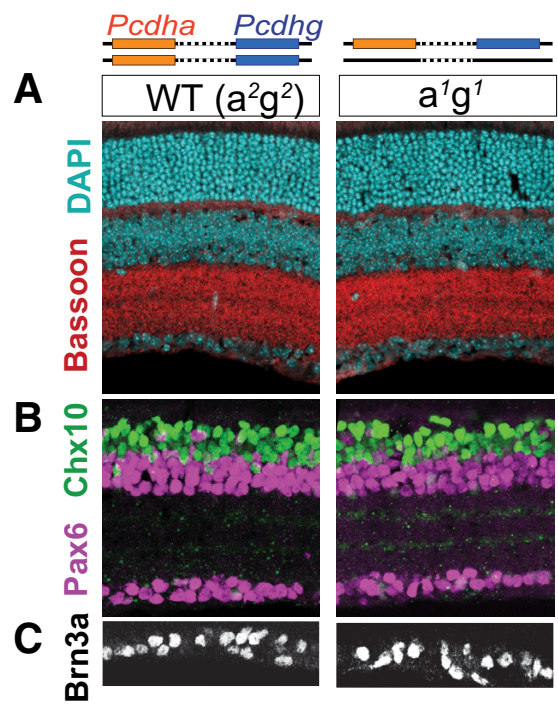

D
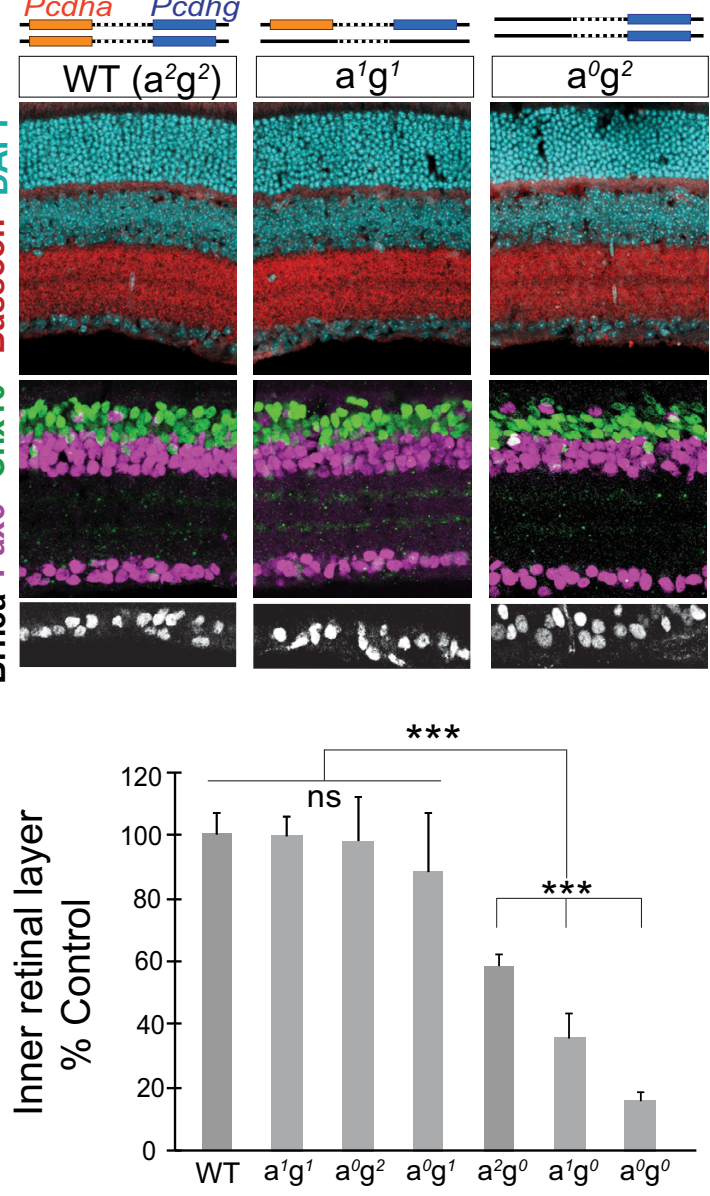
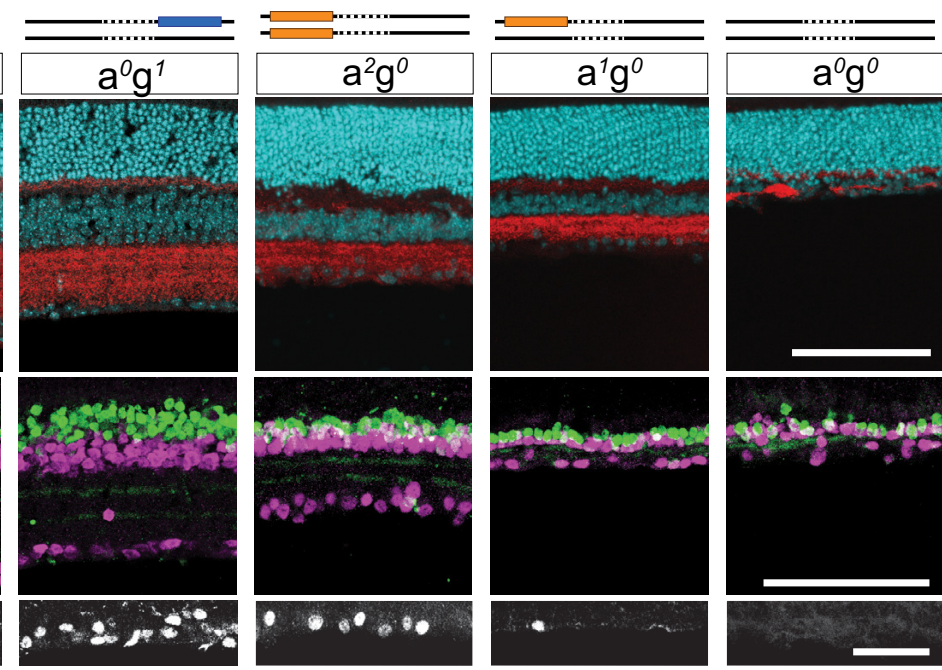

E

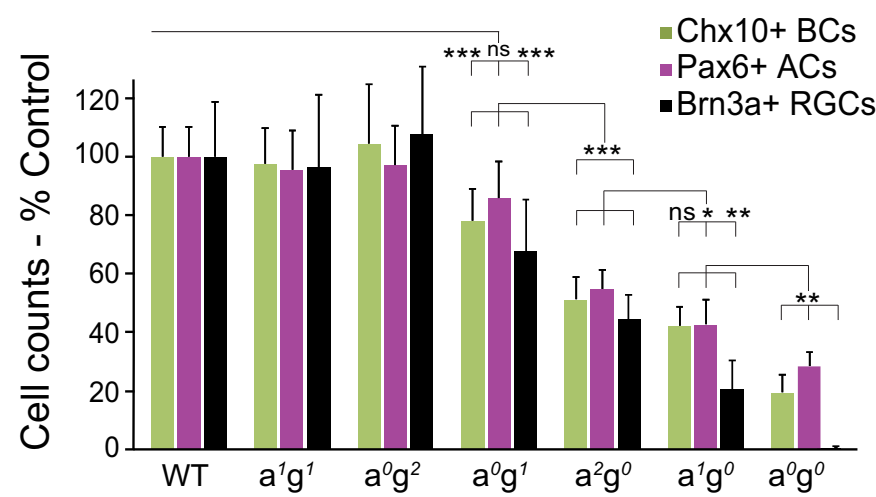

Figure 6. Dose-dependent effects of $P c d h a$ and $P c d h g$ cluster deletions on retinal survival. $\boldsymbol{A}-\boldsymbol{C}$, Top, schematic representation of serial $P c d h a(a$, orange) and $P c d h g(g$, blue) cluster deletions in seven $P$ cdh genotypes. $a^{\mathrm{x}} g^{\mathrm{x}}$ are used to abbreviate the genotypes, where $x$ denotes the number of WT alleles. Successive cluster deletions result in progressive decreases in inner retina layer thickness and cell number. $\boldsymbol{A}$, Retina sections stained with DAPI (cyan) and anti-Bassoon (red). $\boldsymbol{B}, \boldsymbol{C}$, Interneurons and RGCs immunostained for Chx10 $+B C s$ (green), Pax6 + ACs (magenta), and Brn3a + RGCs (white). $\boldsymbol{D}$, Quantifications of inner retina thickness (sum of the INL, IPL, and $\mathrm{GCL}$ ). One-way ANOVA $F_{(8,132)}=182.3, p<0.0001, n=20$ sections, $3-4$ animals per genotype. $\boldsymbol{E}$, Interneuron and RGC cell counts. Chx10+: one-way ANOVA $F_{(9,185)}=99.2, p<0.0001$; Pax6: one-way ANOVA $F_{(9,185)}=96.9, p<0.0001$; Brn3a $+:$ one-way AN0VA $F_{(9,143)}=64.5, p<0.0001$. Bars show means normalized as percentage control sibling data with percentage SD. $n=15$ or 17 images, $4-5$ animals per genotype. ${ }^{* *} p<0.001$, ${ }^{* *} p<0.01,{ }^{*} p<0.05$, pairwise Tukey tests. Scale bars: A, B, $100 \mu \mathrm{m} ; \mathrm{C}, 50 \mu \mathrm{m}$.

sate for the loss of one copy of the Pcdhg cluster $\left(P c d h-a^{1} g^{1}\right)$, providing the minimum threshold of survival activity required for WT levels of interneurons and RGCs. Further comparisons confirmed that the Pcdha cluster partially compensates for the absence of $P c d h g s$ in a dose-dependent manner, as shown by the graded effect of Pcdha cluster copy on enhancing inner retina thickness and cell number $\left(P c d h-a^{2} g^{0}, P c d h-a^{1} g^{0}, P c d h-a^{0} g^{0}\right)$.

We also examined $P c d h-a g^{\mathrm{f} / \mathrm{f}}\left(P c d h-a^{0} g^{\mathrm{f} / \mathrm{f}}\right)$ retinas, in which the targeted $P c d h g^{\mathrm{f}}$ locus remains intact but is hypomorphic. Inner retinal phenotypes trended between those of $P c d h-a^{0} g^{1}$ and $P c d h-a^{2} g^{0}$ retinas. Numbers of bipolar cells and RGCs in $P c d h-a^{0} g^{\mathrm{f} / \mathrm{f}}$ retinas did not differ from those in $P c d h-a^{0} g^{1}$ retinas, but were significantly greater compared to $P c d h-a^{2} g^{0}$ retinas (Chx10+ BCs: one-way ANOVA, $F_{(3,72)}=67.3, p<0.0001$; Pcdh- $a^{0} g^{\mathrm{f} / \mathrm{f}}, 85 \%$ control; Pcdh- $a^{0} g^{1}, 78 \%, p=0.18$ post hoc Tukey test for $a^{2} g^{\mathrm{f} / \mathrm{f}}-a^{0} g^{1} ; P c d h a^{2} g^{0}, 51 \%$ control, $p=0.001$, Tukey test for $a^{0} g^{\mathrm{f} / \mathrm{f}}-a^{2} g^{0}$; Brn3a+ RGCs: one-way ANOVA $F_{(3,62)}=21.7, p<0.0001 ; P c d h-a^{2} g^{\mathrm{f} / \mathrm{f}}, 62 \% ; P c d h-a^{0} g^{1}, 68 \%, p=$ 0.80 , Tukey test; $P c d h-a^{2} g^{0}, 45 \%$ control, $p=0.048$, Tukey test). The inner retinal layers in $P c d h-a^{0} g^{\mathrm{f} / \mathrm{f}}$ retinas were intermediate in thickness between those of $P c d h-a^{0} g^{1}$ and $P c d h-a^{2} g^{0}$ retinas $\left(P c d h-a^{0} g^{\mathrm{f} / \mathrm{f}}, 70 \%\right.$ control; Pcdh- $a^{0} g^{1}, 81 \%, p=0.0053$ Tukey test; $P c d h-a^{2} g^{0}, 58 \%$ control, $p=0.001$ post hoc Tukey test. Oneway ANOVA $\left.F_{(3,72)}=52.2, p<0.0001\right)$. Therefore, the homozygous $P c d h-a g$ allele is approximately functionally equivalent to a heterozygous Pcdhg-null allele.

Together, these data show that, despite the absence of a detectable loss of cells in Pcdha mutant retinas, the Pcdhas cooperate with the Pcdhgs in a dose-dependent fashion to support the survival of retinal interneurons and RGCs.

\section{Pcdhas and Pcdhgs cooperate to mediate}

\section{dendritic self-avoidance}

The radial and uniform distribution of SAC dendrites arises from a process of self-avoidance, whereby sibling dendritic branches repel each other, leading to even coverage of their territory. We showed that Pcdhgs are critical mediators of dendritic selfavoidance in SACs (Lefebvre et al., 2012). Pcdhas are also expressed by SACs (Fig. 1D) and Pcdhbs are broadly expressed in the INL and GCL, suggesting that they may also be involved in SAC dendrite self-avoidance. To test this possibility, we first compared SAC morphology in $P c d h a^{\text {ko }}, P c d h b^{\text {ko }}$, and $P c d h g^{\text {rko }}$ mutants by sparse labeling with a recombinant AAV expressing a membranetagged red fluorescent protein. As reported previously, dendrites 
of single SACs frequently crossed each other and formed loose bundles in Pcdhg ${ }^{\text {rko }}$ mutants (Fig. 7A). In contrast, the morphology of SACs in $P c d h a^{\mathrm{ko}}$ and $P c d h b^{\mathrm{ko}}$ retinas were indistinguishable from that in controls (Fig. 7A).

To assess interactions between Pcdhas and Pcdhgs in SAC dendritic arborization, we initially examined $P c d h-a g^{\text {rko }}$ mutants. Although the number of amacrine cells was reduced by $\sim 72 \%$ in $P c d h-a g^{\text {rko }}$ (Fig. 6E), the number of SACs was reduced by only $\sim 10 \%$ (Fig. $7 B$; unpaired $t$ test, $t_{(32)}=2.0, p=0.059 ; n=17$ regions of interest, 3 animals per genotype), consistent with results from $P c d h g^{\text {rko }}$ mutants ( $\sim 45 \%$ loss of all amacrines (Fig. $6 E$ ), but there was no detectable loss of SACs (Lefebvre et al., 2012)). Nonetheless, we were concerned that interpretation of defects in SAC morphology would be confounded by the loss of their synaptic partners and by the collapse of the two SAC strata (Fig. 7C). To circumvent this problem, we deleted Pcdhgs selectively from SACs using a ChAT-cre line $\left(P c d h-a g^{\mathrm{f} / \mathrm{f}} ; C h A T^{\mathrm{cre}}\right.$ or $\left.P c d h-a g^{\text {SACko }}\right)$. Compared with $P c d h-a g^{\text {rko }}$ retinas, the stratification of SAC processes was preserved in $P c d h-a g^{\text {SACko }}$ retinas, although some gaps were present (Fig. $7 C$ ). The inner retina was also preserved in $P c d h-a g^{\text {SACko }}$ because inner retina layer thickness and numbers of RGCs were indistinguishable from control $P c d h-a g^{\mathrm{f} / \mathrm{f}}$ retinas (INL-GCL layers: $P c d h-a g^{\text {SACko }}, 73.5 \pm 1.8$ $\mu \mathrm{m} ;$ Pcdh- $a g^{\mathrm{f} / \mathrm{f}}, 70.7 \pm 2.2 \mu \mathrm{m} ; n=12$ sections from $3-4$ animals; unpaired $t$ test, $t_{(22)}=0.98, p=0.34$. Brn3a+ RGCs: $P c d h$ $a g^{\text {SACko }}, 14.8$ cells/area $\pm 0.8 ; P c d h-a g^{\mathrm{f} / \mathrm{f}}, 15.7$ cells/area \pm 1.1 ; $n=15$ sections from 3-4 animals; unpaired $t$ test, $t_{(28)}=0.68$, $p=0.50)$. Moreover, terminals of amacrine and bipolar subtypes segregated to appropriate IPL sublaminae (Fig. 7D), similar to WT (see Fig. 5A,C). Therefore, deletion of Pcdhgs in SACs in a Pcdh- $a^{\text {ko }}$ null background had no detectable non-cell-autonomous effects.

When viewed in whole mount, the SAC dendritic plexus was clearly disorganized in $P c d h-a g^{\text {SACko }}$ retinas. Defects were more severe in this genotype than in single $P c d h g^{\text {rko }}$ and $P c d h g^{\text {SACko }}$ mutants. Pcdha ${ }^{\text {ko }}$ mutant retinas were unaffected. Conversely, SAC dendrites were more dramatically disorganized in double $P c d h-a g^{\text {rko }}$ retinas than in $P c d h-a g^{\text {SACko }}$ mutants (Fig. $7 E, F$ ). Given the absence of non-cell-autonomous effects in $P c d h$ - $a g$ SACko retinas, we conclude that the more striking defects in Pcdh-ag rko SACs result from non-cell-autonomous effects and therefore used $P c d h-a g^{\text {SACko }}$ for further studies of SAC morphology.

Individually labeled $P c d h-a g^{\text {SACko }}$ SACs displayed dendrite crossing and bundling defects that were dramatically more pronounced than those in Pcdhg mutants. The dendrites formed larger fascicles and, in the majority of cases, they lost radial symmetry (Fig. $7 G$ ). The collapse was so severe that it was infeasible to count individual branches or crossings as we had done for Pcdhg mutants (Lefebvre et al., 2012). We therefore devised an alternative method to quantify the difference between $P c d h g^{\text {SACko }}$ and $P c d h-a g{ }^{\text {SACko }}$ SACs by measuring radial symmetry. Examples are shown in Figure $8, A$ and $B$. Whereas the radial symmetry of SAC arbors was degraded to a small but significant extent in $P c d h g^{\text {SACko }}$ retinas, it was nearly abolished in $P c d h-a g^{\text {SACko }}$ retinas (Fig. $8 C$; one-way ANOVA, $F_{(3,64)}=42.1, p<0.0001$, Tukey's test, $p<$ 0.0001 , except for WT- $\mathrm{a}^{2} \mathrm{~g}^{0}, p=0.042 ; n=17$ SACs, $2-4$ animals from genotype). Moreover, the radius of the SAC arbor did not differ significantly between WT and $P c d h g^{\text {SACko }}$ retinas, but was significantly decreased in $P c d h$ - $a g$ SACko (Fig. $8 D$; one-way ANOVA, $F_{(3,64)}=13.4, p<0.0001$; Tukey's test, $p<0.0001$, except for WT-a $\left.{ }^{2}{ }^{0}, p=0.80\right)$. We also analyzed SACs from Pcdhg; Pcdh$a g^{\text {SACko }}$ transheterozygous mice, which retained one copy of the Pcdha cluster $\left(a^{1} g^{0}\right)$. SAC defects were intermediate in severity between those of $P c d h g$ and $P c d h$-ag mutants (Fig. $8 A-D$ ), reveal- ing the dose-dependent contributions of Pcdhas. Therefore, Pcdhas and Pcdhgs cooperate to mediate dendritic self-avoidance.

\section{Pcdhas and Pcdhgs make equal contributions to dendrite self-avoidance in Purkinje cells}

Pcdhgs are required for dendritic self-avoidance in cerebellar Purkinje cells and in SACs (Lefebvre et al., 2012). Because Purkinje cells stochastically express subsets of both Pcdha and Pcdhg isoforms (Esumi et al., 2005; Kaneko et al., 2006), we investigated whether Pcdhas affect the patterning of Purkinje cells dendrites either alone or in combination with Pcdhgs. We labeled individual Purkinje cells in Pcdha ${ }^{\mathrm{ko}}$ and WT animals by injection of AAV encoding GFP into neonatal mice. Deletion of the Pcdhas led to self-avoidance defects, including branch overlaps and overall disorganization of branching (Fig. 9A,B). Self-crossing dendrites were distinguished by branch intersections detected in single confocal planes and close apposition of branches in orthogonal views (Fig. 9 $A^{\prime}, B^{\prime}$ ). The severity and rate of self-crossing defects in $P c d h-a^{\text {ko }}$ mutants were similar to those in $P c d h-g^{\text {PCko }}$ mutants (PCko: with Purkinje-specific Cre driver, Pcp2-cre) (Fig. 9C, $C^{\prime}$ ) (Lefebvre et al., 2012). Therefore, in contrast to SACs, in which Pcdhas have no discernable effect on their own, the Pcdha and $P c d h g$ clusters have similar functions in dendrite self-avoidance of Purkinje cells.

Finally, we deleted both Pcdhas and Pcdhgs from Purkinje cells by injecting AAV vectors encoding Cre and a Cre-dependent fluorescent reporter into perinatal $P c d h-a g^{\mathrm{f} / \mathrm{f}}$ mutants $\left(P c d h-a g^{\text {PCko }}\right)$. In the absence of both clusters, Purkinje cells developed a dramatic excess of dendritic branches and a greater frequency of branch self-overlaps (570\%, normalized as percentage of branch overlaps in control littermates) compared with single $P c d h-a^{\text {ko }}$ (252\%, $p<0.0001$, Mann-Whitney test) or Pcdh-g ${ }^{\text {PCko }}$ Purkinje cells $\left(245 \%, p<0.0001\right.$, Mann-Whitney test; Fig. 9D, $\left.D^{\prime}, E\right)$. Although we cannot exclude non-cell-autonomous effects of constitutive Pcdha deletions in $P c d h a^{\text {ko }}$ and $P c d h$-ag mutant cerebellums, double $P c d h-a g^{\text {PCko }}$ Purkinje cells were generated by sparse deletion of Pcdhg and these cells revealed a significant interaction between Pcdhas and Pcdhgs in dendritic defects. We noted that arbor area was significantly reduced in double mutant Purkinje cells ( $p=0.002$, Mann-Whitney test), whereas those of single mutant Purkinje cells were unaffected $\left(P c d h-a^{\text {ko }}, p=0.37\right.$ and $P c d h-g^{\text {PCko }}, p=0.89$, Mann-Whitney test; Fig. $9 F$ ). In contrast, when viewed along the $y-z$ axis (parasagittal plane), the arbors of $P c d h-a g^{\text {PCko }}$ cells occupied increased space compared with control, with multiple sets of overlapping of dendrites spanning the entire arbor (Fig. 9G,H). The multiplanar arrangement of $P c d h-a g^{\text {PCko }}$ Purkinje dendrites differed qualitatively from the monoplanar arbor formed by control Purkinje cells (Fig. 9I, J; Kaneko et al., 2011). Therefore, deletion of both $P c d h a$ and $P c d h g$ clusters affects multiple aspects of Purkinje arbor organization, including area and monoplanarity.

Together, these findings reveal that the Pcdhas and Pcdhgs serve equal roles in Purkinje cells and that Pcdh diversity provided by both clusters is essential for optimal dendrite patterning. Importantly, they also show that the Pcdhas are not generally subordinate in effect to Pcdhgs, but differ in their contributions in a cell-type-specific manner. The dose-dependent and cell-typespecific contributions of Pcdhas and Pcdhgs in retinal survival and SAC and Purkinje dendrite self-avoidance are summarized in Table 1. 
A
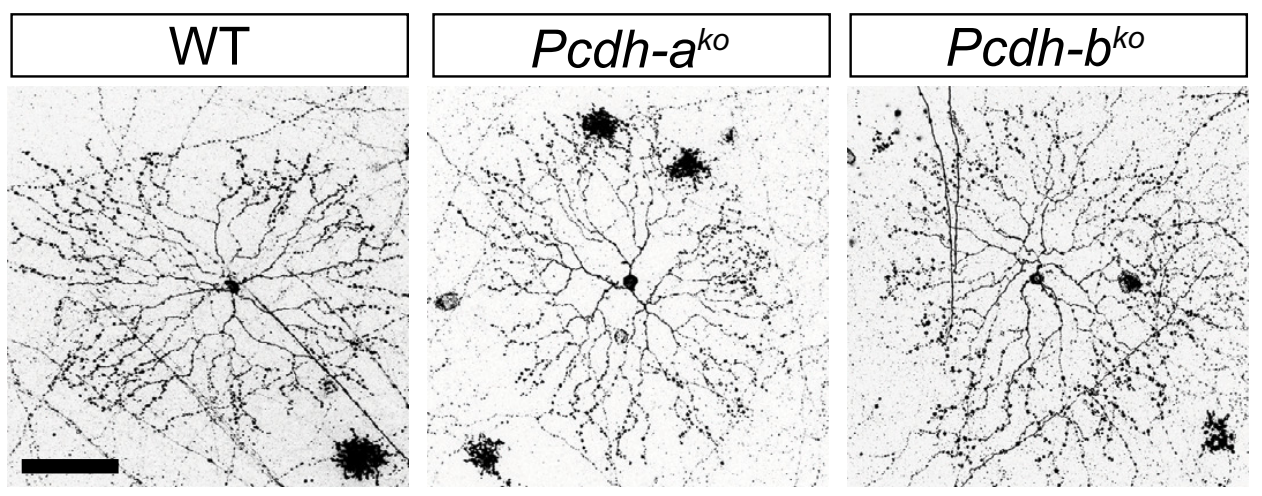

B
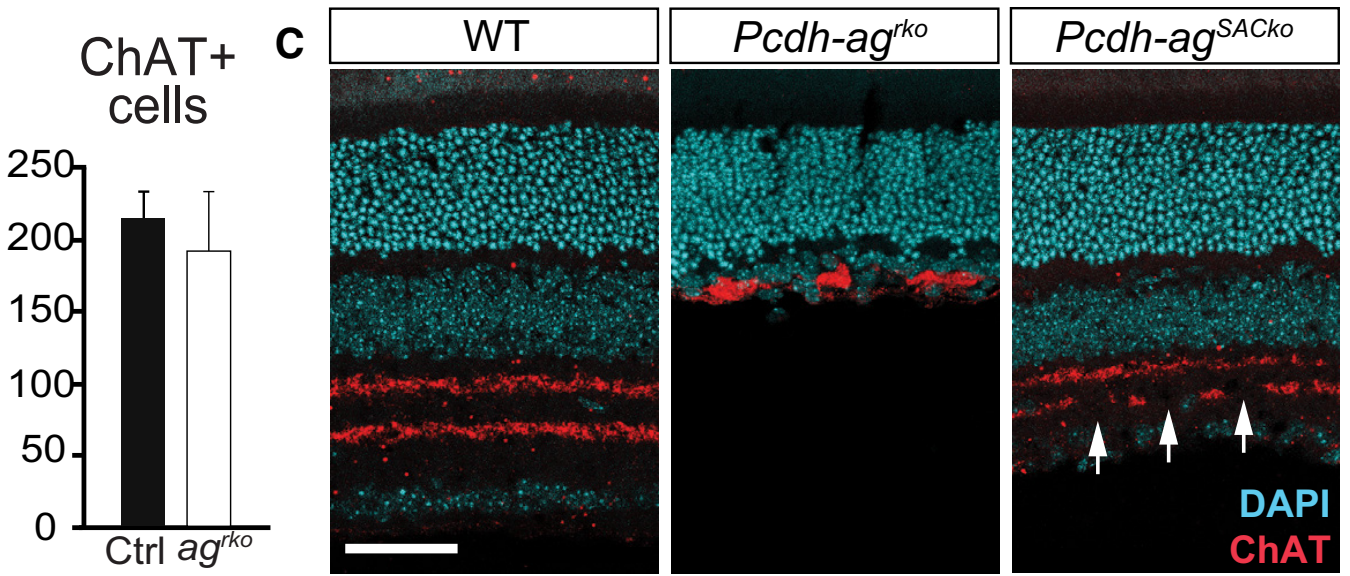

D
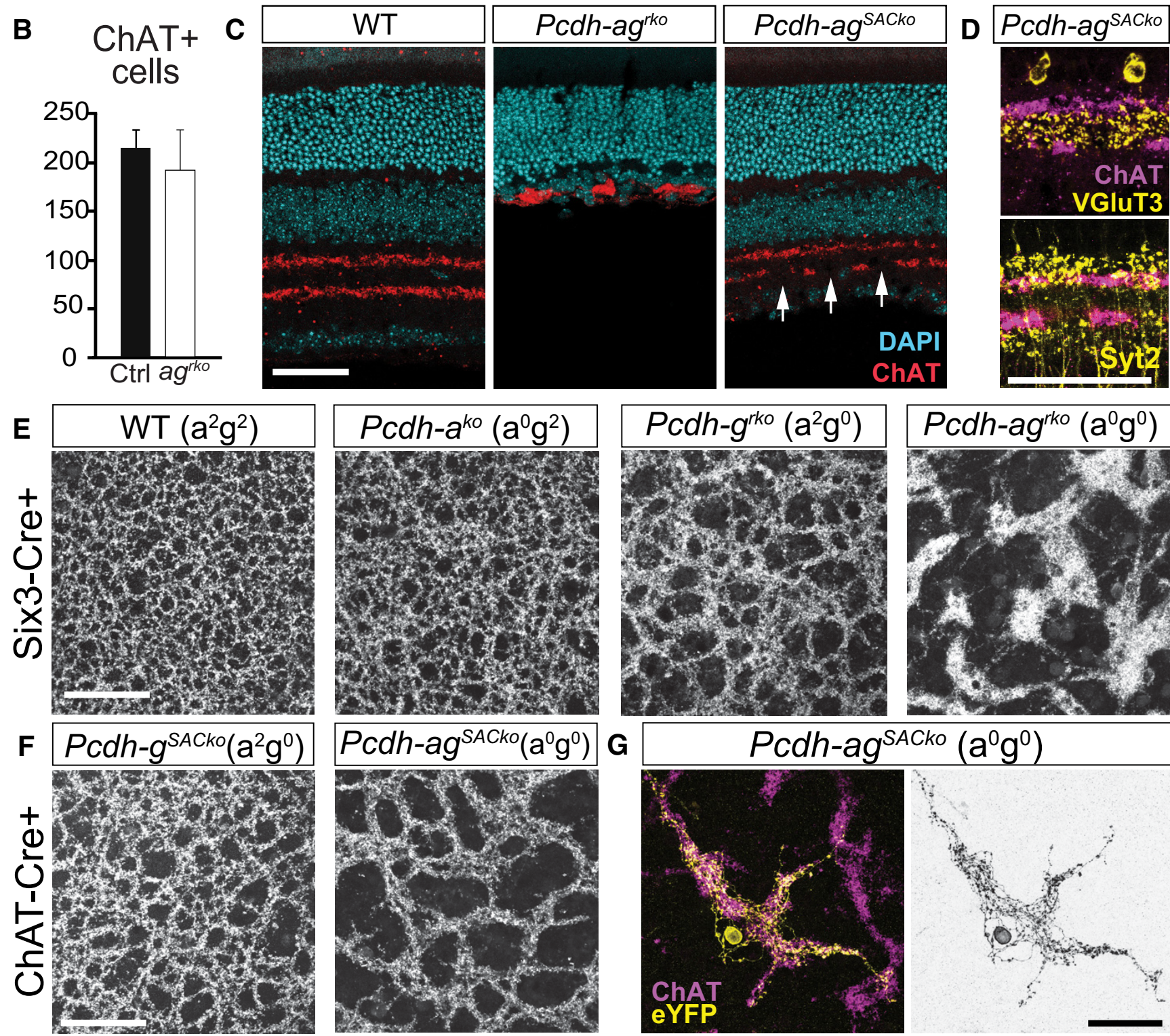

Figure 7. The $P c d h a$ and $P c d h g$ clusters cooperate to promote dendrite self-avoidance. $A$, Single SACs labeled with AAV-membrane-RFP in WT, $P c d h a^{\text {ko }}, P c d h b^{\text {ko }}$, and $P c d h g^{\text {rko }}$ retinal whole mounts. $B$, Numbers of ChAT + SACs in WT and $P c d h-a g^{\text {rko }}$ retinas. Bars show means \pm SEM. $n=17$ areas were analyzed, 3 animals per genotype, Student's $t$ test, $t_{(32)}=2.0, p=0.059$. $C, S A C$ dendritic layers in WT, $P c d h-a g^{\text {rko }}$, and $P c d h-a g^{\text {SACko }}\left[P c d h-a^{\text {conexldel; }} g^{f} x\right.$ Chat-cre] retinas labeled with DAPI (cyan) and anti-ChAT (red). Arrows indicate gaps in the SAC layers despite preservation of the inner retina in Pcdh-ag ${ }^{\text {SACko }}$ retina. D, Laminar segregation of vGluT3 + AC processes (yellow, top) and Syt2 + BC terminals (yellow, bottom) is intact in Pcdh-ag ${ }^{\text {SACko }}$ retina (ChAT + SAC layers, magenta). $\boldsymbol{E}, \boldsymbol{F}$, Whole-mount retina preparation showing ChAT-labeled plexus formed by SAC dendrites in WT, single $P c d h-a^{\mathrm{ko}}$ and $P c d h-g^{\text {rko }}$ mutants, and double $P c d h-a g^{\text {rko }}$ mutants $(\boldsymbol{E})$ and SAC-specific $P c d h-g{ }^{\text {SACko }}$ and $P c d h-a g^{\text {SACko }}$ mutants $(\boldsymbol{F})$. Note larger gaps and increased fasciculation of SAC plexus in double mutant $P c d h$-ag compared with WT or single $P c d h g$ mutant retinas. G, Morphology of a single SAC from Pcdh-ag ${ }^{\text {SACko }}$ retina labeled with eYFP (yellow) and SAC plexus labeled with anti-ChAT (magenta). Scale bars, $50 \mu \mathrm{m}$. 
A
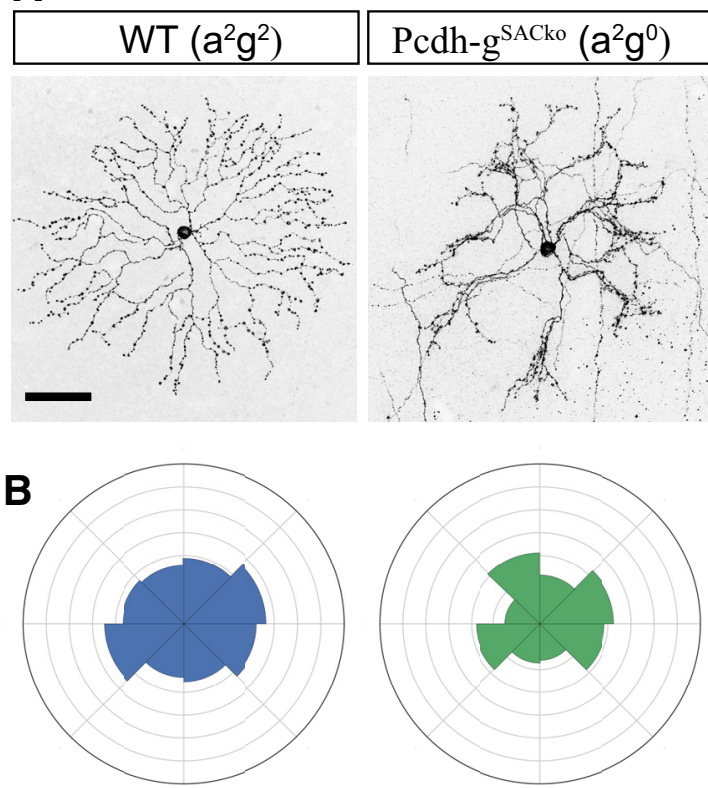

C
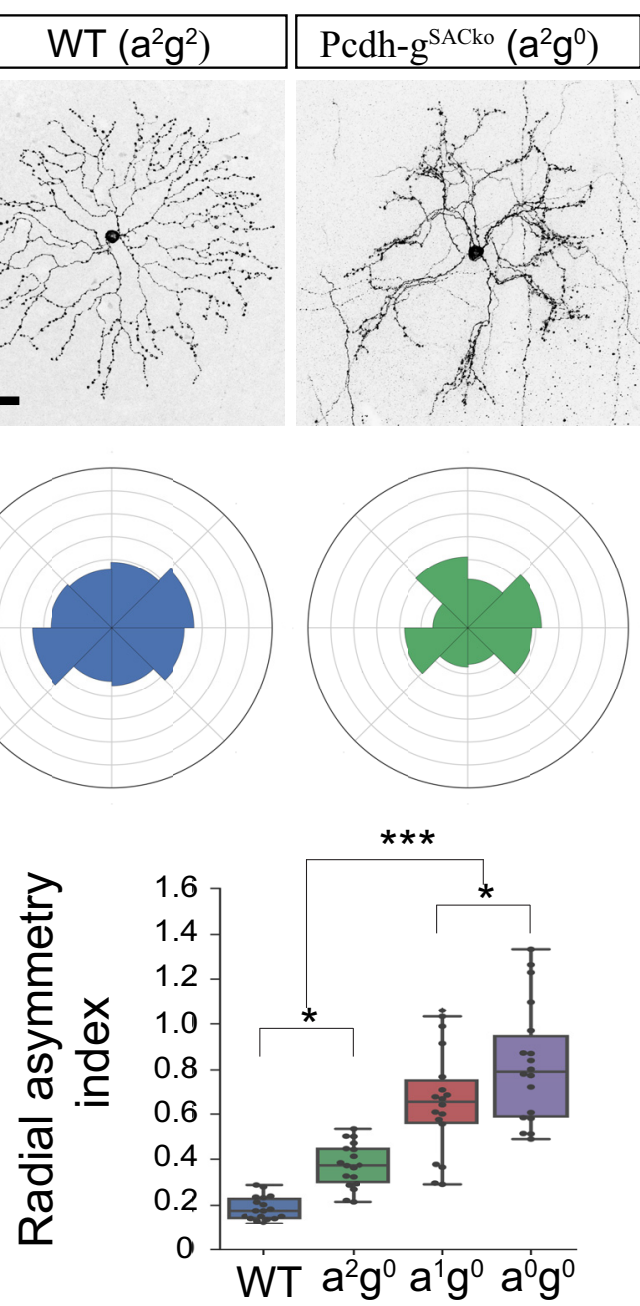
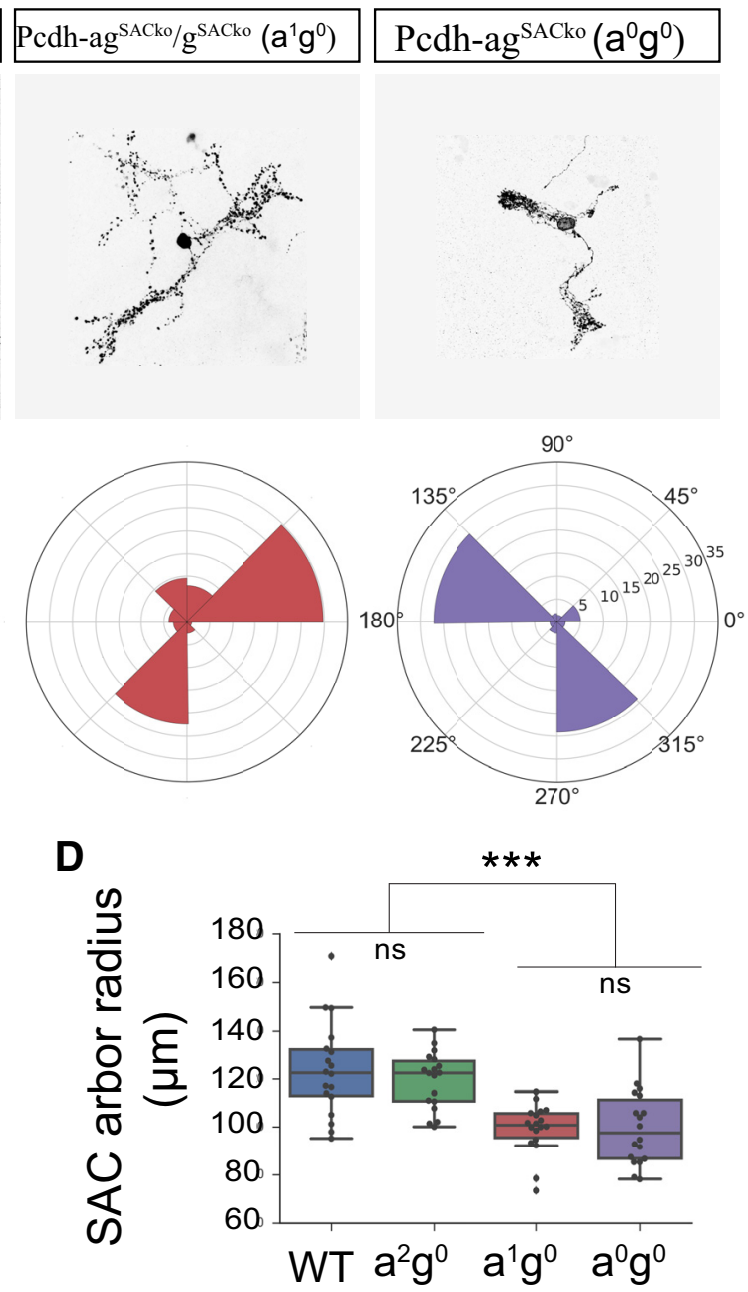

Figure 8. Dose-dependent interactions between $P c d h a s$ and $P$ cdhys clusters on $S A C$ dendrite arborization and self-avoidance. $A$, Morphology of single $S A C s$ reveal effects of removing a single $\left(a^{1} g^{0}\right)$ or both $P$ cdha clusters $\left(a^{0} g^{0}\right)$ on dendrite self-avoidance in SACs lacking Pcdhgs $\left(P c d h-g^{\text {SACko }}\right)$. B, Polar plot representations for SACs in $\boldsymbol{A}$ showing the pixel distribution of dendrites across eight polar segments. $\boldsymbol{C}$, Analysis of SAC polar plots such as those in $\boldsymbol{B}$ using an asymmetry index, which reports on the SDs from expected radial symmetry (index 0.0 ) in which pixels are equally

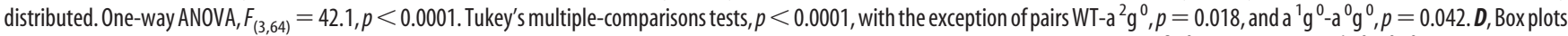
showing SAC dendritic field radius. One-way ANOVA, $F_{(3,64)}=13.4, p<0.0001$. Tukey's multiple-comparisons tests, $p<0.0001$. Pairs WT- ${ }^{2} g^{0}, p=0.80$, and $a^{1} g^{0}$ - ${ }^{0} g^{0}, p=0.90$ are not significant (ns). $\boldsymbol{C}, \boldsymbol{D}, n=17 \mathrm{SACs}, 2-4$ animals from genotype. ${ }^{* *} p<0.0001,{ }^{*} p<0.05$. Scale bars, $50 \mu \mathrm{m}$.

\section{Discussion}

The clustered Pcdhs have the potential to generate extraordinary cell surface diversity and specificity for patterning the nervous system. They do so by combinatorial interactions among Pcdh members: each Pcdh exhibits homophilic binding in trans, but multimerizes promiscuously in cis. Selective trans binding between matching combinations can then greatly expand the recognition specificity presented by Pcdh multimers (Schreiner and Weiner, 2010; Thu et al., 2014; Nicoludis et al., 2015; Rubinstein et al., 2015; Goodman et al., 2016; Rubinstein et al., 2017). Here, we demonstrate that the Pcdhas and Pcdhgs interact to promote neuronal survival in retina and dendritic self-avoidance in both retina and cerebellum. However, interactions between the clusters differ between these tissues (Table 1). In retina, loss of Pcdhas has no obvious consequence, but defects in $P c d h a / P c d h g$ double mutants are dramatically more severe than those of Pcdhg mutants. In contrast, defects in dendritic patterning of cerebellar Purkinje cells are significant in both Pcdha and Pcdhg mutants and markedly more severe in $P c d h a / P c d h g$ double mutants. These patterns have been called unequal and partial redundancy, respectively (Briggs et al., 2006; Barbaric et al., 2007).

\section{Unequal redundancy of Pcdhas and Pcdhgs in retinal development}

Duplicated genes can play redundant, overlapping, or completely distinct roles. For the Pcdh clusters, there are at least three factors relevant to their relationships. First, the selective homophilic specificity of Pcdh trans interactions implies that Pcdha and Pcdhg proteins can mediate different intercellular interactions. Second, Pcdhas and Pcdhgs have different intracellular domains and could therefore engage distinct signaling pathways. Third, expression patterns of Pcdha and Pcdhg genes differ because of distinct regulatory elements (Ribich et al., 2006; Noguchi et al., 2009; Kehayova et al., 2011; Yokota et al., 2011). Determining the extent of redundancy among Pcdh clusters has been challenging due to the complexity of the locus and the presence of essential genes. Recent studies of double and triple Pcdh cluster mutants from two groups have revealed redundant and cooperative func- 

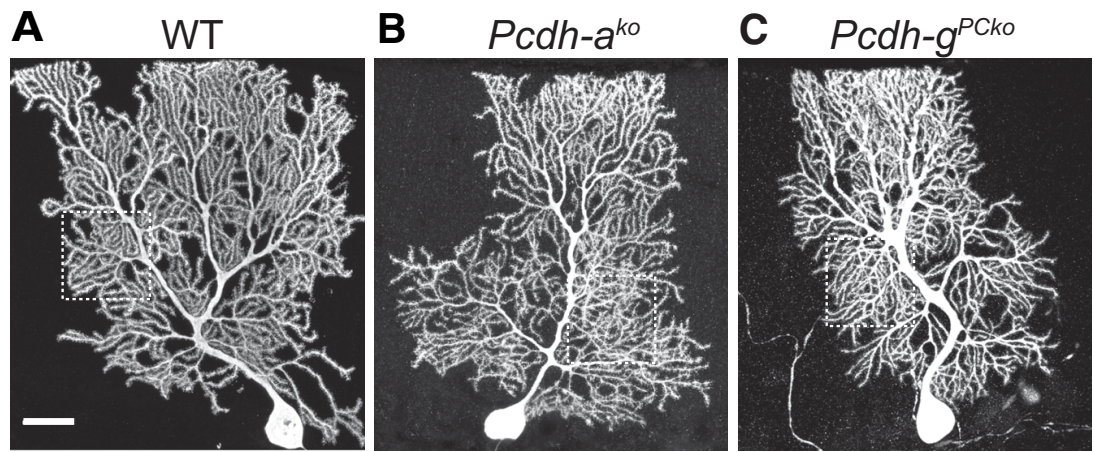

D Pcdh-ag ${ }^{\text {PCko }}$
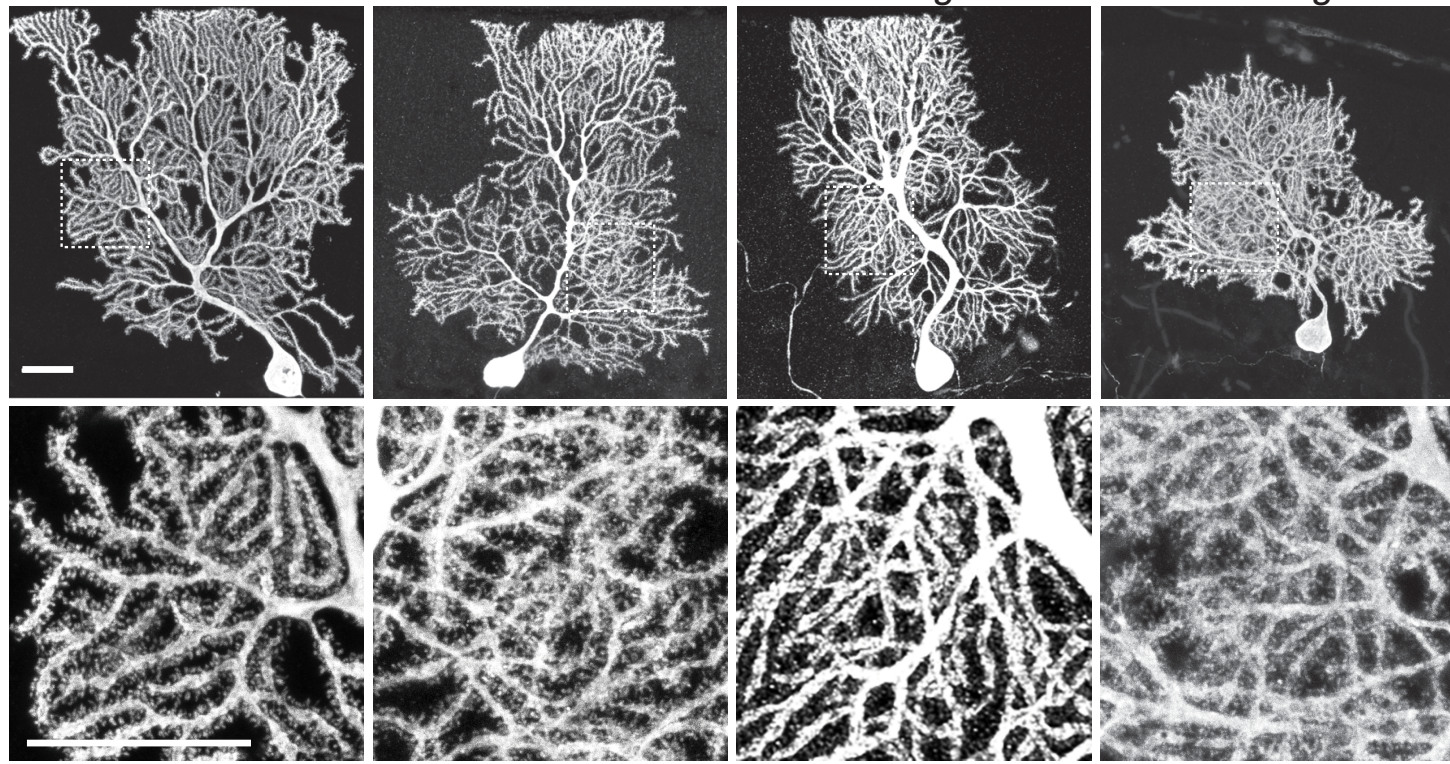

$\mathbf{A}^{\prime}$

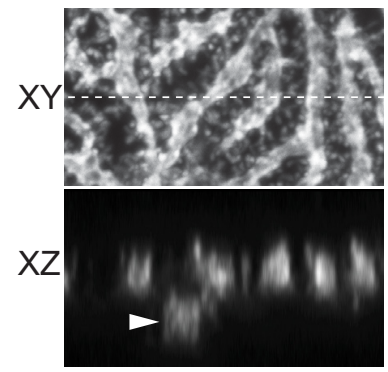

B'

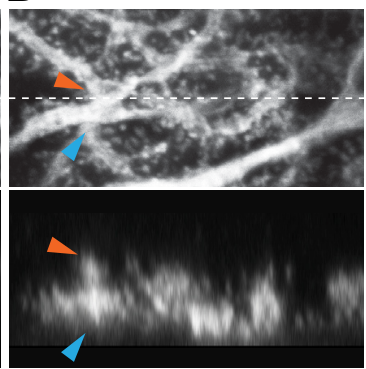

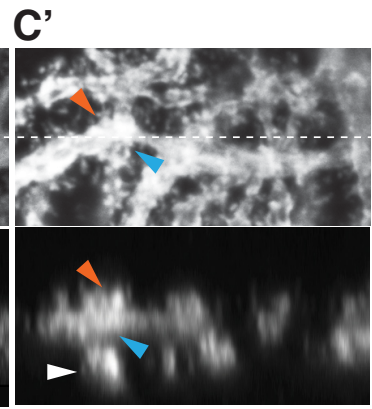

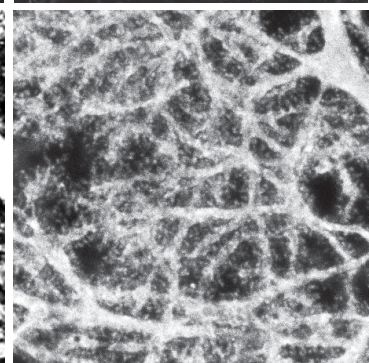

E

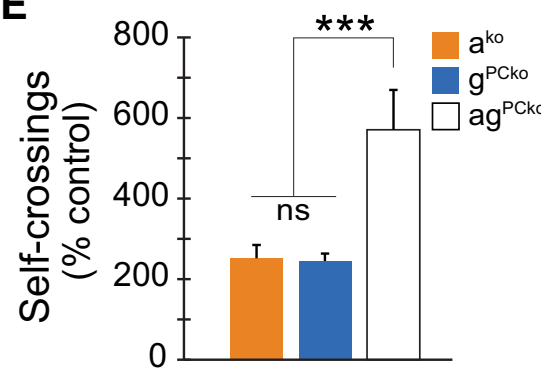

$\mathbf{F}$

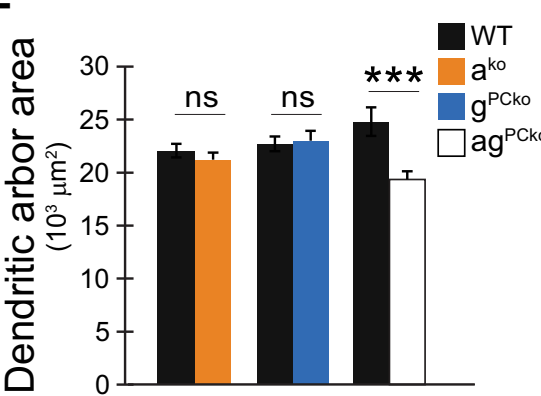

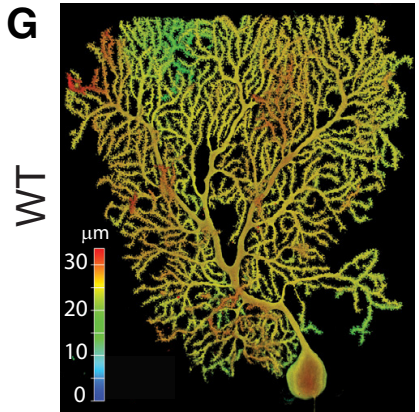

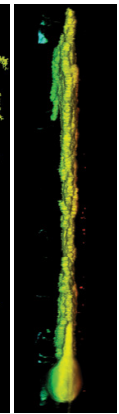

H

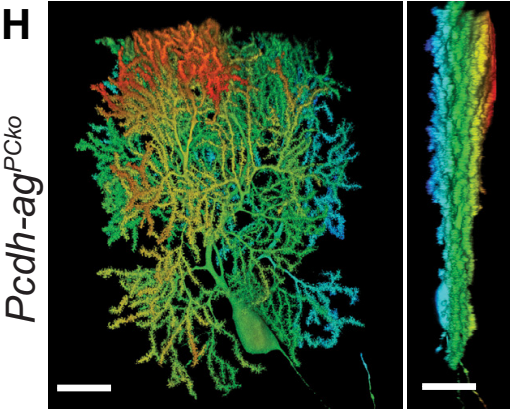

D'

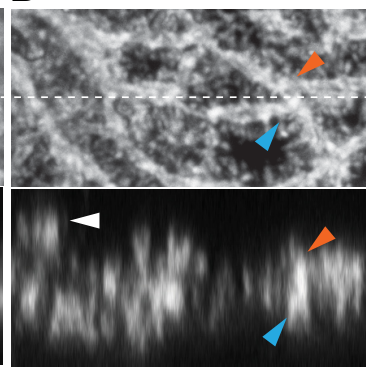

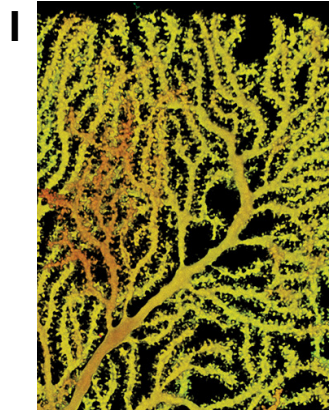

J

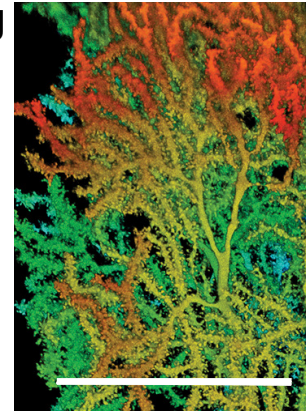

Figure 9. Pcdhas are essential for dendrite self-avoidance of Purkinje cells and serve equal functions with Pcdhgs. $\boldsymbol{A}-\boldsymbol{D}$, Morphology of single mature (P35) cerebellar Purkinje cells labeled with AAV-GFP or AAV-RFP. Areas boxed in top panels are shown at higher magnification in bottom. Purkinje cells lacking $P$ cdha, $P$ cdhg, or both clusters display disorganized arborizations marked by frequent self-crossing defects, but defects are more dramatic in double than single mutants. Double mutant Purkinje cells also exhibit increased branching and smaller dendritic territories. $\boldsymbol{A}^{\prime}-\boldsymbol{D}^{\prime}$, Orthogonal $x-y$ (top) and $x-z$ (bottom) views of dendrite self-crossings in high-magnification confocal stacks. Blue and orange arrowheads mark intersecting branches in the $x-y$ plane (maximum projection of substack; dashed line is plane for $x-z$ sectioning) and their close apposition in $x-z$ (note merged fluorescent signals from two branches). "Overpassing" branches that extend in distant planes are individually resolved in $x-z$ (white arrowheads) and are not counted as self-crossings. Single and double Pcdh mutant Purkinje cells display increased self-crossings and overpassing branches compared with WT. $\boldsymbol{E}$, Quantifications of dendrite self-crossings detected in $70 \times 70 \mu \mathrm{m}$ areas from z-stack confocal images. Bars show (Figure legend continues.) 
Table 1. Summary of $P$ cdh cluster dosage relationships in retina and Purkinje cell development

\begin{tabular}{|c|c|c|c|c|}
\hline Pcdh clusters & Genotype & $\begin{array}{l}\text { Inner } \\
\text { retina cell } \\
\text { survival }\end{array}$ & $\begin{array}{l}\text { SAC } \\
\text { dendrite } \\
\text { self-avoidance }\end{array}$ & $\begin{array}{l}\text { Purkinje } \\
\text { dendrite } \\
\text { self-avoidance }\end{array}$ \\
\hline \multirow{3}{*}{$\begin{array}{l}\text { Pcdh } a^{2} g^{2} \\
a^{1} g^{1}\end{array}$} & Pcdh- $a^{\text {wt }} ; g^{\text {wt }}$ & - & - & - \\
\hline & Pcdh-ag ${ }^{+/ c k o}$ & & & \\
\hline & (rko, Six3-cre; PCko, Pcp2-cre) & - & - & - \\
\hline$a^{0} g^{2}$ & $P c d h-a^{\text {ko } / \mathrm{ko}}$ & - & - & $x$ \\
\hline$a^{0} g^{1}$ & $P c d h-a^{\mathrm{ko}} /-a g^{c k o}$ & $x$ & nd & nd \\
\hline$a^{2} g^{0}$ & Pcdh-g $g^{\text {cko /cko }}$ & $\times \times$ & $\times \times$ & $x$ \\
\hline$a^{1} g^{0}$ & $P c d h-a g^{\mathrm{cko}} /-g^{c k o}$ & $x \times x$ & $\times \times \times \times$ & nd \\
\hline$a^{0} g^{0}$ & Pcdh-ag cko/cko & $x \times x \times$ & $X \times X \times X$ & $\times \times$ \\
\hline $\begin{array}{l}\text { SAC-specific } \\
a^{2} g^{0}\end{array}$ & Pcdh-g ${ }^{\text {SACko /ko }}$ (Chat-Cre) & - & $x$ & \\
\hline$S A C-a^{1} g^{0}$ & Pcdh-ag ${ }^{\text {SACko }} /-g^{\text {SACko }}$ & - & $\times \times$ & \\
\hline$S A C-a^{0} g^{0}$ & Pcdh-ag SACko/SACko & - & $\times \times \times \times$ & \\
\hline
\end{tabular}

Phenotype score: -, unaffected; $\times$, disrupted and graded by severity $(\times n)$; nd, not determined.

tions in multiple aspects of embryonic CNS development (Hasegawa et al., 2016; Mountoufaris et al., 2017). To study Pcdh cluster diversity in the postnatal CNS, we generated a double Pcdha;Pcdhg mutant allele in which Pcdhg could be inactivated conditionally, preserving postnatal viability. By comparing Pcdh cluster expression and mutant retinas lacking one or both clusters, we discovered both overlapping and distinct features of Pcdhas and Pcdhgs in retinal development. Our principal results are as follows.

First, although Pcdhas and Pcdhgs (as well as Pcdhbs) are broadly expressed in the inner retina, removal of the Pcdhgs led to loss of RGCs, bipolar cells, and amacrine cells (Lefebvre et al., 2008), whereas removal of Pcdhas had no detectable effect on their survival. In contrast, removal of both Pcdha and Pcdhg clusters enhanced the Pcdhg phenotype, unmasking shared roles for the Pcdhas in survival of inner retinal cells.

Second, Pcdhas expression is strikingly higher in RGCs that in other retinal cells and survival of RGCs is more dramatically affected than survival of bipolar or amacrine cells in Pcdh-ag mutants. These patterns suggest a unique role of Pcdhas in RGC development, but the nature of this role is unclear. Pcdhas are required for organizing RGC axons in the lateral geniculate nucleus (Meguro et al., 2015). Roles of Pcdhgs in this process remained to be determined, but it is intriguing to speculate that the devastating loss of RGCs in Pcdha/Pcdhg double mutants might result in part from loss of target-derived support consequent to target defects in double mutants.

Third, Pcdhas and Pcdhgs show unequally redundant roles of the morphogenesis of SAC dendrites. In Pcdhg mutants, dendritic

(Figure legend continued.) means \pm SEM normalized as percentage of quantifications from control siblings; $n=10-15$ cells, two-way ANOVA, ${ }^{* * *} p<0.001$. $\boldsymbol{F}$, Area of dendritic arborizations. Bars show mean \pm SEM. $n=15-17$ cells per genotype. Purkinje arbor area in $P c d h a^{\mathrm{ko}}$ and $P c d h g^{\mathrm{PCk}}$ are not significantly different from control $(p=0.37$ and $p=0.89)$. $P c d h-a{ }^{P C k o}$ arbors are significantly reduced in size compared with littermate controls ( $p=$ 0.003 , Mann-Whitney $U$ test) and when compared with the combined pool of controls ${ }^{* * *} p=$ 0.0004, Mann-Whitney $U$ test). Data on Pcdhg mutants in $\boldsymbol{E}$ and $\boldsymbol{F}$ are from Lefebvre et al. (2012). G, H, Depth representation of WT and Pcdh-ag ${ }^{\text {PCko }}$ Purkinje arborization with color spectrum code depicting positions in $z$ (sagittal planes). Left, $x-y$ view of depth-coded 3D rendering of Purkinje cells. Right, $y-z$ rotation (parasagittal planes). Arbors of WT Purkinje cells are confined to narrow sagittal planes. Dendrites of $P c d h-a g^{\text {PCko }}$ Purkinje cells arborize into multiple planes spanning nearly the entire depth-spectrum. $I, J$, High-magnification view of monoplanar arrangement of WT dendritic arbors and multiplanarity of $P\left(d h-a g^{P C k o}\right.$ arbors. Note light blue branches beneath the red-colored dendrites (white arrow). Scale bars, $25 \mu \mathrm{m}$. branches fail to repel each other, a phenomenon called selfavoidance, resulting in loss of the radially symmetrical morphology (Lefebvre et al., 2012). We detected no self-avoidance defects in Pcdha mutants, but found a striking exacerbation of the Pcdhg phenotype in double mutants. Our previous analysis demonstrated that restoration of a single Pcdhg is sufficient to rescue self-avoidance in Pcdhg cluster mutants, but that expression of multiple Pcdhgs is required for self-/non-self-discrimination in which SAC dendrites can synapse on dendrites of other SACs while avoiding sibling dendrites (Lefebvre et al., 2012; Kostadinov and Sanes, 2015). Analysis of self-/non-self-discrimination in $P c d h a / P c d h g$ double mutants is a promising means of probing the relationship of redundancy between entire clusters to redundancy among isoforms.

Fourth, Pcdhas partially compensate for loss of Pcdhgs in a dose-dependent manner, revealing interactions between Pcdhas and Pcdhgs in retinal survival and SAC dendrite self-avoidance. Similarly in spinal cord, redundant but unequal roles were demonstrated for the Pcdha and Pcdhb clusters in survival (Hasegawa et al., 2016). Increased apoptosis was also noted in embryonic retinas from triple $P c d h$ - $a b g^{\text {ko }}$ (Hasegawa et al., 2016), but analysis was limited due to the immaturity of retinal cell types at this stage.

Fifth, threshold levels of Pcdh activity are required for survival and self-avoidance. In the absence of Pcdhas, a single Pcdhg cluster was haploinsufficient for survival, but addition of a Pcdha cluster restored cell number to WT levels. Together with dosedependent contributions of Pcdhas, these data demonstrate that the Pcdhas signal with Pcdhgs to promote survival. By extension, we surmise that subthreshold levels of Pcdh activity in brainstem and spinal circuits contributes to the neonatal lethality frequently encountered among $P c d h-a g^{\mathrm{f}}$ mutants.

Divergent Pcdha and Pcdhg activities could arise from differences in expression or in biochemical or signaling properties associated with their distinct cytoplasmic regions. Our finding that the Pcdhas only partially compensate for RGC survival despite high expression levels suggests that differences in expression levels are unlikely to account for differences in survival. Given the crucial roles for the three C-type Pcdhgs isoforms in survival (Chen et al., 2012), the Pcdha-dependent contributions to survival could be mediated by the two C-type Pcdhas, which are more closely related to the C-type Pcdhgs than to the alternate Pcdhas.

\section{Partial redundancy of Pcdhas and Pcdhgs in cerebellar Purkinje cells}

In contrast to SACs, Purkinje neurons are equally dependent on the Pcdhas and Pcdhgs for dendrite self-avoidance. Removal of either Pcdh cluster results in modest but clear phenotypes, whereas dual inactivation of the clusters dramatically enhanced Purkinje dendrite arborization defects. These findings reflect a partial redundancy for Pcdhas and the Pcdhgs. Therefore, the Pcdhas are not necessarily the weaker paralogs and Pcdh cluster diversity is essential for neuronal patterning of some cell types. Moreover, our results indicate that functional interactions between Pcdh clusters will differ among cell types or contexts.

Interestingly, the dendritic phenotypes in double $P c d h$ mutant SAC and Purkinje cells were qualitatively different. Pcdh-ag mutant Purkinje cells were multiplanar with an excess of branches. This phenotype might arise from retraction defects, which is consistent with live-imaging studies showing retraction of Purkinje dendrites upon contact with sibling (self) branches (Fujishima et al., 2012). Fujishima et al. (2012) also showed that self-contacts 
and retractions occur at terminal branch tips, which is also the site of branch addition. Pcdhs might therefore couple dendrite self-recognition with elimination in Purkinje cells to limit branch number and overlap. Whereas dendrite-dendrite interactions within a cell (self-avoidance) are degraded in single $P c d h$ mutants, dendrite-dendrite interactions between cells (termed tiling) may also be comprised in double mutants. Conversely, $P c d h$-ag mutant SACs displayed increased fasciculation between sibling dendrites, which severely distorted the radial organization. SAC dendrites tend to cofasciculate (Lefebvre et al., 2012), which is critical for establishing reciprocal connections with neighboring SACs, but it must be minimized among sibling dendrites (Kostadinov and Sanes, 2015). Although further analyses are required to determine the underlying mechanisms, our observations raise the possibility that Pcdhs mediate self-avoidance in mammalian neurons through diverse cellular processes such as branch elimination and repulsion.

\section{Overlapping but distinct roles for Pcdhs in neuronal development}

Two recent studies have demonstrated cooperation among Pcdh clusters in the embryonic olfactory system. Olfactory sensory neurons (OSNs) terminate onto shared glomeruli in the olfactory bulb. In triple $P c d h$-abg ${ }^{\mathrm{ko}}$ mutant neonates, OSN reached their target but collapsed and failed to elaborate branches (Mountoufaris et al., 2017). Glomerular defects were more severe in triple $P c d h-a b g$ ko neonates than in single cluster mutants (Hasegawa et al., 2016; Mountoufaris et al., 2017). Our results extend this analysis to postnatal development and demonstrate interactions between clusters in two other regions: retina and cerebellum. In contrast, patterning of sensory la afferents terminals in spinal cord are only dependent on the Pcdhg cluster (Prasad and Weiner, 2011; Hasegawa et al., 2016). Therefore, functional and redundant interactions between Pcdh clusters differ among cell types and developmental pathways.

Other, related studies have shown both shared and distinct roles of isoforms within the Pcdha and Pcdhg clusters (see Introduction) (Chen et al., 2012; Lefebvre et al., 2012; Kostadinov and Sanes, 2015; Chen et al., 2017). The picture emerging from these analyses is that diversity of Pcdh isoforms both within and between clusters is critically important for neural development, with both isoforms and clusters mediating overlapping yet distinct functions. With a large, functionally redundant ensemble of molecules, the clustered Pcdhs can mediate highly selective events when there are numerous potential interactions and ensure robustness during patterning of complex neuronal structures.

\section{References}

Barbaric I, Miller G, Dear TN (2007) Appearances can be deceiving: phenotypes of knockout mice. Brief Funct Genomic Proteomic 6:91-103. CrossRef Medline

Briggs GC, Osmont KS, Shindo C, Sibout R, Hardtke CS (2006) Unequal genetic redundancies in arabidopsis-a neglected phenomenon? Trends Plant Sci 11:492-498. CrossRef Medline

Cai D, Cohen KB, Luo T, Lichtman JW, Sanes JR (2013) Improved tools for the Brainbow toolbox. Nat Methods 10:540-547. CrossRef Medline

Chen WV, Alvarez FJ, Lefebvre JL, Friedman B, Nwakeze C, Geiman E, Smith C, Thu CA, Tapia JC, Tasic B, Sanes JR, Maniatis T (2012) Functional significance of isoform diversification in the protocadherin $\gamma$ gene cluster. Neuron 75:402-409. CrossRef Medline

Chen WV, Nwakeze CL, Denny CA, O'Keeffe S, Rieger MA, Mountoufaris G, Kirner A, Dougherty JD, Hen R, Wu Q, Maniatis T (2017) Pcdhalphac2 is required for axonal tiling and assembly of serotonergic circuitries in mice. Science 356:406-411. CrossRef Medline

Cong L, Ran FA, Cox D, Lin S, Barretto R, Habib N, Hsu PD, Wu X, Jiang W,
Marraffini LA, Zhang F (2013) Multiplex genome engineering using CRISPR/Cas systems. Science 339:819-823. CrossRef Medline

Cui Q, Ren C, Sollars PJ, Pickard GE, So KF (2015) The injury resistant ability of melanopsin-expressing intrinsically photosensitive retinal ganglion cells. Neuroscience 284:845-853. CrossRef Medline

Duan X, Qiao M, Bei F, Kim IJ, He Z, Sanes JR (2015) Subtype-specific regeneration of retinal ganglion cells following axotomy: effects of osteopontin and mTOR signaling. Neuron 85:1244-1256. CrossRef Medline

Esumi S, Kakazu N, Taguchi Y, Hirayama T, Sasaki A, Hirabayashi T, Koide T, Kitsukawa T, Hamada S, Yagi T (2005) Monoallelic yet combinatorial expression of variable exons of the protocadherin-alpha gene cluster in single neurons. Nat Genet 37:171-176. CrossRef Medline

Fujishima K, Horie R, Mochizuki A, Kengaku M (2012) Principles of branch dynamics governing shape characteristics of cerebellar Purkinje cell dendrites. Development 139:3442-3455. CrossRef Medline

Furuta Y, Lagutin O, Hogan BL, Oliver GC (2000) Retina- and ventral forebrain-specific cre recombinase activity in transgenic mice. Genesis 26:130-132. CrossRef Medline

Garrett AM, Weiner JA (2009) Control of CNS synapse development by $\{\gamma\}$-protocadherin-mediated astrocyte-neuron contact. J Neurosci 29: 11723-11731. CrossRef Medline

Garrett AM, Schreiner D, Lobas MA, Weiner JA (2012) $\gamma$-protocadherins control cortical dendrite arborization by regulating the activity of a FAK/ PKC/MARCKS signaling pathway. Neuron 74:269-276. CrossRef Medline

Gegonne A, Tai X, Zhang J, Wu G, Zhu J, Yoshimoto A, Hanson J, Cultraro C, Chen QR, Guinter T, Yang Z, Hathcock K, Singer A, Rodriguez-Canales J, Tessarollo L, Mackem S, Meerzaman D, Buetow K, Singer DS (2012) The general transcription factor TAF7 is essential for embryonic development but not essential for the survival or differentiation of mature $\mathrm{T}$ cells. Mol Cell Biol 32:1984-1997. CrossRef Medline

Goodman KM, Rubinstein R, Thu CA, Bahna F, Mannepalli S, Ahlsén G, Rittenhouse C, Maniatis T, Honig B, Shapiro L (2016) Structural basis of diverse homophilic recognition by clustered alpha- and beta-protocadherins. Neuron 90:709-723. CrossRef Medline

Hasegawa S, Hamada S, Kumode Y, Esumi S, Katori S, Fukuda E, Uchiyama Y, Hirabayashi T, Mombaerts P, Yagi T (2008) The protocadherin-alpha family is involved in axonal coalescence of olfactory sensory neurons into glomeruli of the olfactory bulb in mouse. Mol Cell Neurosci 38:66-79. CrossRef Medline

Hasegawa S, Kumagai M, Hagihara M, Nishimaru H, Hirano K, Kaneko R, Okayama A, Hirayama T, Sanbo M, Hirabayashi M, Watanabe M, Hirabayashi T, Yagi T (2016) Distinct and cooperative functions for the protocadherin-alpha, -beta and - $\gamma$ clusters in neuronal survival and axon targeting. Front Mol Neurosci 9:155. CrossRef Medline

Hasegawa S, Kobayashi H, Kumagai M, Nishimaru H, Tarusawa E, Kanda H, Sanbo M, Yoshimura Y, Hirabayashi M, Hirabayashi T, Yagi T (2017) Clustered protocadherins are required for building functional neural circuits. Front Mol Neurosci 10:114. CrossRef Medline

Hirano K, Kaneko R, Izawa T, Kawaguchi M, Kitsukawa T, Yagi T (2012) Single-neuron diversity generated by protocadherin-beta cluster in mouse central and peripheral nervous systems. Front Mol Neurosci 5:90. CrossRef Medline

Kaneko M, Yamaguchi K, Eiraku M, Sato M, Takata N, Kiyohara Y, Mishina M, Hirase H, Hashikawa T, Kengaku M (2011) Remodeling of monoplanar purkinje cell dendrites during cerebellar circuit formation. PLoS One 6:e20108. CrossRef Medline

Kaneko R, Kato H, Kawamura Y, Esumi S, Hirayama T, Hirabayashi T, Yagi T (2006) Allelic gene regulation of pcdh-alpha and pcdh- $\gamma$ clusters involving both monoallelic and biallelic expression in single purkinje cells. J Biol Chem 281:30551-30560. CrossRef Medline

Katori S, Hamada S, Noguchi Y, Fukuda E, Yamamoto T, Yamamoto H, Hasegawa S, Yagi T (2009) Protocadherin-alpha family is required for serotonergic projections to appropriately innervate target brain areas. J Neurosci 29:9137-9147. CrossRef Medline

Kay JN, Chu MW, Sanes JR (2012) MEGF10 and MEGF11 mediate homotypic interactions required for mosaic spacing of retinal neurons. Nature 483:465-469. CrossRef Medline

Kehayova P, Monahan K, Chen W, Maniatis T (2011) Regulatory elements required for the activation and repression of the protocadherin-alpha gene cluster. Proc Natl Acad Sci U S A 108:17195-17200. CrossRef Medline

Kostadinov D, Sanes JR (2015) Protocadherin-dependent dendritic selfavoidance regulates neural connectivity and circuit function. eLife 4 . 
Lefebvre JL (2017) Neuronal territory formation by the atypical cadherins and clustered protocadherins. Semin Cell Dev Biol 69:111-121. CrossRef Medline

Lefebvre JL, Zhang Y, Meister M, Wang X, Sanes JR (2008) $\gamma$-protocadherins regulate neuronal survival but are dispensable for circuit formation in retina. Development 135:4141-4151. CrossRef Medline

Lefebvre JL, Kostadinov D, Chen WV, Maniatis T, Sanes JR (2012) Protocadherins mediate dendritic self-avoidance in the mammalian nervous system. Nature 488:517-521. CrossRef Medline

Lefebvre JL, Sanes JR, Kay JN (2015) Development of dendritic form and function. Annu Rev Cell Dev Biol 31:741-777. CrossRef Medline

Meguro R, Hishida R, Tsukano H, Yoshitake K, Imamura R, Tohmi M, Kitsukawa T, Hirabayashi T, Yagi T, Takebayashi H, Shibuki K (2015) Impaired clustered protocadherin-alpha leads to aggregated retinogeniculate terminals and impaired visual acuity in mice. J Neurochem 133:66-72. CrossRef Medline

Molumby MJ, Keeler AB, Weiner JA (2016) Homophilic protocadherin cell-cell interactions promote dendrite complexity. Cell Rep 15:10371050. CrossRef Medline

Mountoufaris G, Chen WV, Hirabayashi Y, O'Keeffe S, Chevee M, Nwakeze CL, Polleux F, Maniatis T (2017) Multicluster pcdh diversity is required for mouse olfactory neural circuit assembly. Science 356:411-414. CrossRef Medline

Nicoludis JM, Lau SY, Schärfe CP, Marks DS, Weihofen WA, Gaudet R (2015) Structure and sequence analyses of clustered protocadherins reveal antiparallel interactions that mediate homophilic specificity. Structure 23:2087-2098. CrossRef Medline

Noguchi Y, Hirabayashi T, Katori S, Kawamura Y, Sanbo M, Hirabayashi M, Kiyonari H, Nakao K, Uchimura A, Yagi T (2009) Total expression and dual gene-regulatory mechanisms maintained in deletions and duplications of the pcdha cluster. J Biol Chem 284:32002-32014. CrossRef Medline

Passini MA, Wolfe JH (2001) Widespread gene delivery and structurespecific patterns of expression in the brain after intraventricular injections of neonatal mice with an adeno-associated virus vector. J Virol 75:1238212392. CrossRef Medline

Pérez de Sevilla Müller L, Sargoy A, Rodriguez AR, Brecha NC (2014) Melanopsin ganglion cells are the most resistant retinal ganglion cell type to axonal injury in the rat retina. PLoS One 9:e93274. CrossRef Medline

Prasad T, Weiner JA (2011) Direct and indirect regulation of spinal cord ia afferent terminal formation by the $\gamma$-protocadherins. Front Mol Neurosci 4:54. CrossRef Medline

Prasad T, Wang X, Gray PA, Weiner JA (2008) A differential developmental pattern of spinal interneuron apoptosis during synaptogenesis: insights from genetic analyses of the protocadherin- $\gamma$ gene cluster. Development 135:4153-4164. CrossRef Medline

Ribich S, Tasic B, Maniatis T (2006) Identification of long-range regulatory elements in the protocadherin-alpha gene cluster. Proc Natl Acad Sci U S A 103:19719-19724. CrossRef Medline

Rodriguez AR, de Sevilla Müller LP, Brecha NC (2014) The RNA binding protein RBPMS is a selective marker of ganglion cells in the mammalian retina. J Comp Neurol 522:1411-1443. CrossRef Medline
Rossi J, Balthasar N, Olson D, Scott M, Berglund E, Lee CE, Choi MJ, Lauzon D, Lowell BB, Elmquist JK (2011) Melanocortin-4 receptors expressed by cholinergic neurons regulate energy balance and glucose homeostasis. Cell Metab 13:195-204. CrossRef Medline

Rubinstein R, Thu CA, Goodman KM, Wolcott HN, Bahna F, Mannepalli S, Ahlsen G, Chevee M, Halim A, Clausen H, Maniatis T, Shapiro L, Honig B (2015) Molecular logic of neuronal self-recognition through protocadherin domain interactions. Cell 163:629-642. CrossRef Medline

Rubinstein R, Goodman KM, Maniatis T, Shapiro L, Honig B (2017) Structural origins of clustered protocadherin-mediated neuronal barcoding. Semin Cell Dev Biol 69:140-150. CrossRef Medline

Sanes JR, Zipursky SL (2010) Design principles of insect and vertebrate visual systems. Neuron 66:15-36. CrossRef Medline

Schreiner D, Weiner JA (2010) Combinatorial homophilic interaction between $\gamma$-protocadherin multimers greatly expands the molecular diversity of cell adhesion. Proc Natl Acad Sci U S A 107:14893-14898. CrossRef Medline

Su H, Marcheva B, Meng S, Liang FA, Kohsaka A, Kobayashi Y, Xu AW, Bass $J$, Wang X (2010) $\gamma$-protocadherins regulate the functional integrity of hypothalamic feeding circuitry in mice. Dev Biol 339:38-50. CrossRef Medline

Thu CA, Chen WV, Rubinstein R, Chevee M, Wolcott HN, Felsovalyi KO, Tapia JC, Shapiro L, Honig B, Maniatis T (2014) Single-cell identity generated by combinatorial homophilic interactions between alpha, beta, and $\gamma$ protocadherins. Cell 158:1045-1059. CrossRef Medline

Toyoda S, Kawaguchi M, Kobayashi T, Tarusawa E, Toyama T, Okano M, Oda M, Nakauchi H, Yoshimura Y, Sanbo M, Hirabayashi M, Hirayama T, Hirabayashi T, Yagi T (2014) Developmental epigenetic modification regulates stochastic expression of clustered protocadherin genes, generating single neuron diversity. Neuron 82:94-108. CrossRef Medline

Wang X, Weiner JA, Levi S, Craig AM, Bradley A, Sanes JR (2002) $\gamma$ protocadherins are required for survival of spinal interneurons. Neuron 36 : 843-854. CrossRef Medline

Weiner JA, Wang X, Tapia JC, Sanes JR (2005) $\gamma$ protocadherins are required for synaptic development in the spinal cord. Proc Natl Acad Sci U S A 102:8-14. CrossRef Medline

Wu Q, Maniatis T (1999) A striking organization of a large family of human neural cadherin-like cell adhesion genes. Cell 97:779-790. CrossRef Medline

Wu Q, Zhang T, Cheng JF, Kim Y, Grimwood J, Schmutz J, Dickson M, Noonan JP, Zhang MQ, Myers RM, Maniatis T (2001) Comparative DNA sequence analysis of mouse and human protocadherin gene clusters. Genome Res 11:389-404. CrossRef Medline

Yokota S, Hirayama T, Hirano K, Kaneko R, Toyoda S, Kawamura Y, Hirabayashi M, Hirabayashi T, Yagi T (2011) Identification of the cluster control region for the protocadherin-beta genes located beyond the protocadherin- $\gamma$ cluster. J Biol Chem 286:31885-31895. CrossRef Medline

Young RW (1984) Cell death during differentiation of the retina in the mouse. J Comp Neurol 229:362-373. CrossRef Medline

Zipursky SL, Sanes JR (2010) Chemoaffinity revisited: dscams, protocadherins, and neural circuit assembly. Cell 143:343-353. CrossRef Medline 